دراسة اقتصادية للوضع الإنتاجي و التجارة الخارجية لأهم للنباتات الطبية و العطرية فى مصر حسن موسى رضوان المعهد العالى للتعاون و الإرشاد الزر اعى بأسيوط

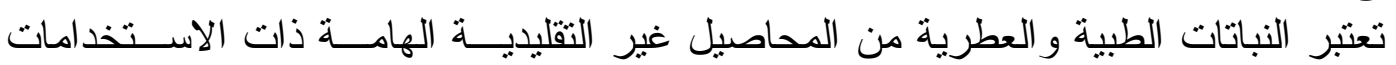

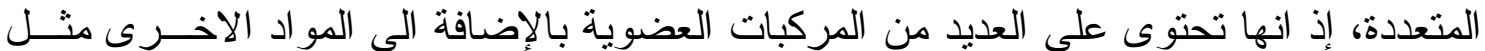

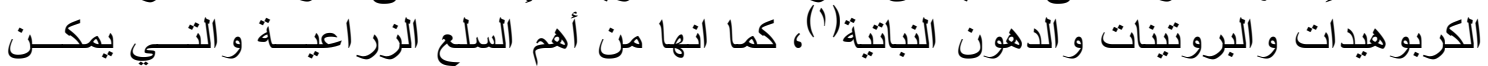

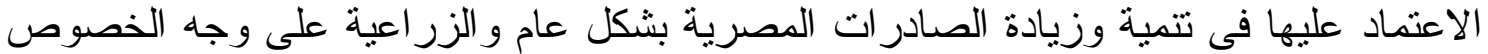

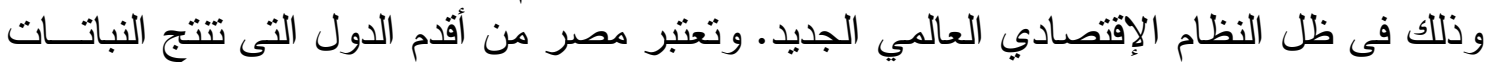

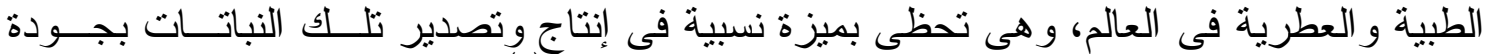

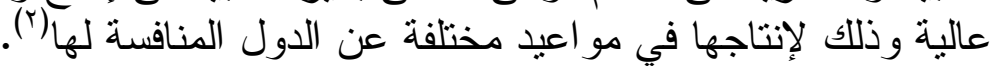

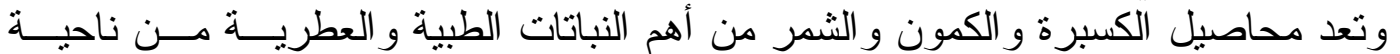

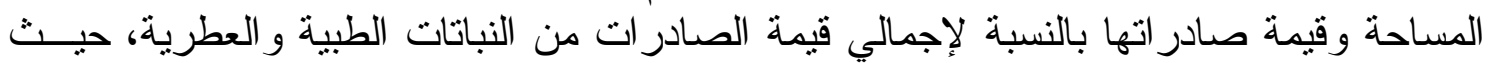

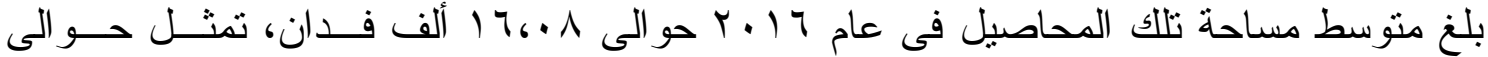

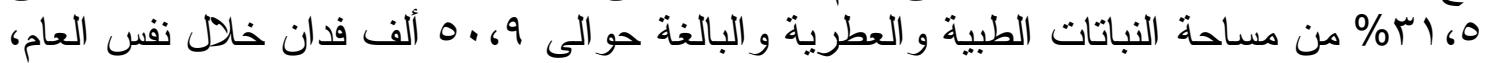

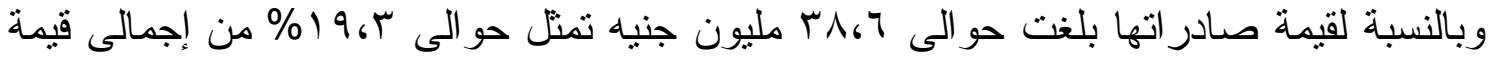

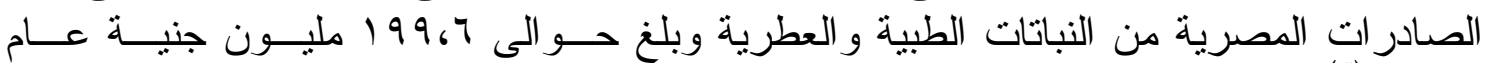

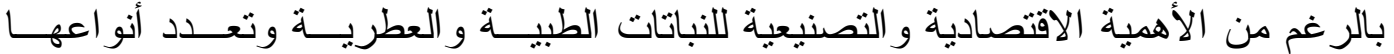

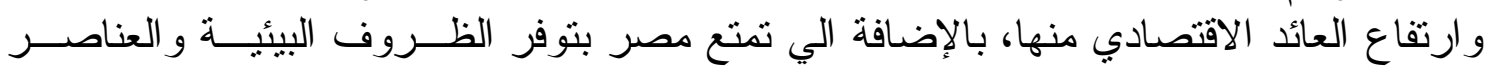

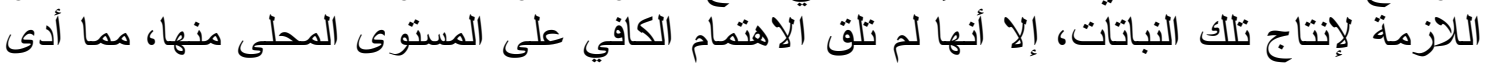

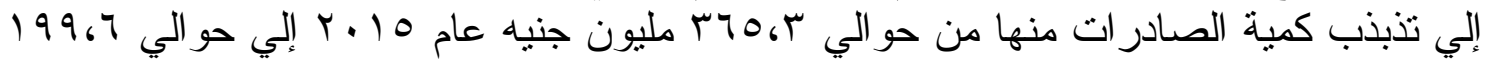

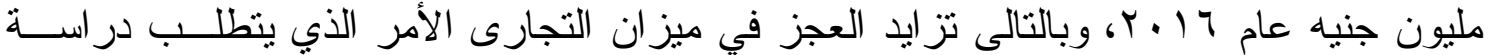

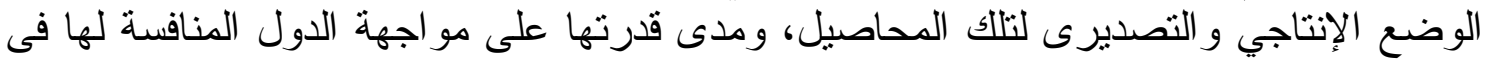

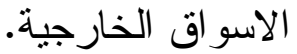

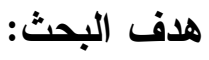

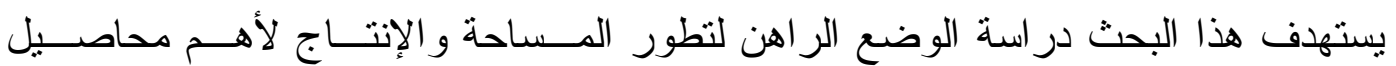

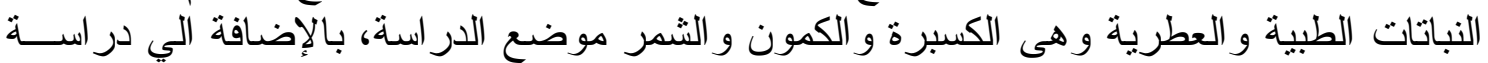

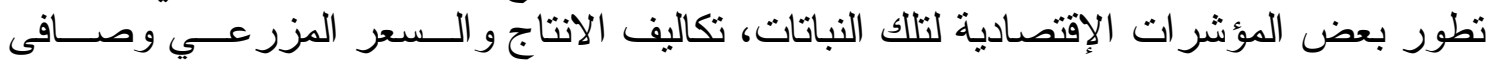

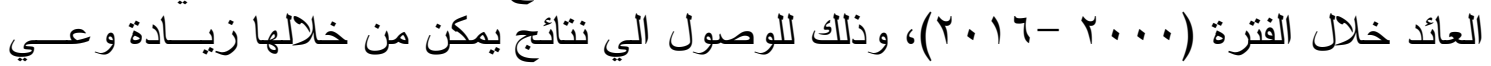
المنتجين وبالتالي التوسع في هذا الإنتاج، وكذللك در اسة تطور كمية وقيمة الصـادر ات المــصرية

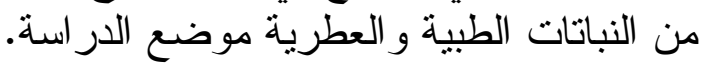

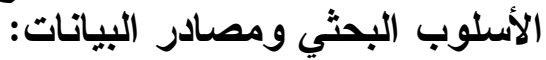

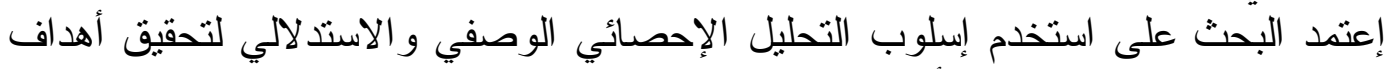

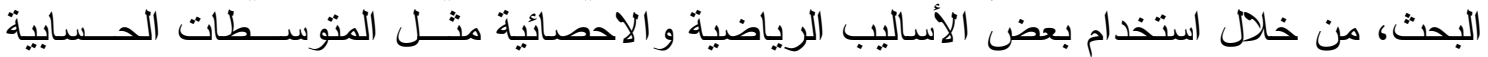

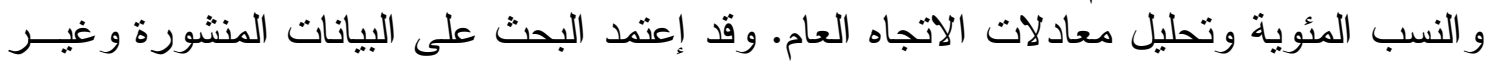

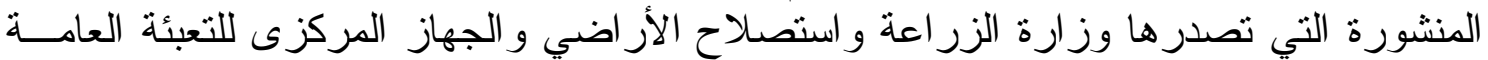
و الإحصاء، بالإضافة الي بعض المر اجع و الدر اسات السابقة و التى تخدم الخدم هذا البحث. 
أولأ: تطور البحت المساحة والإتتاج الكتي لأهم النباتات الطبية والعطرية فى مــصر خــلال الفتـرة 1- محصول الكسبرة :

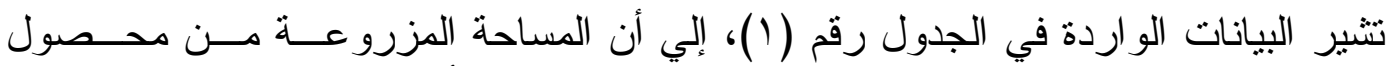

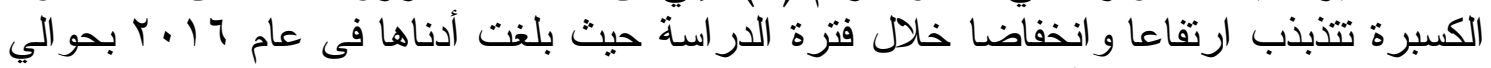

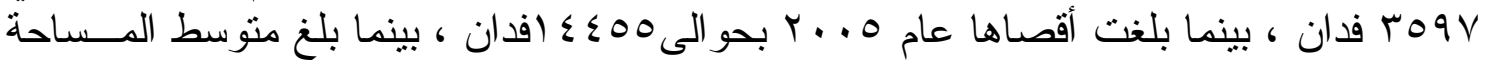

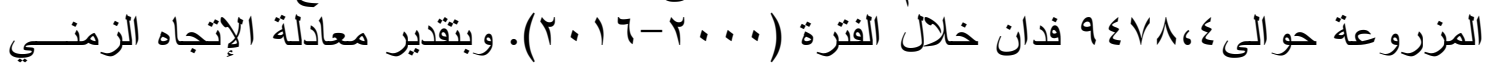

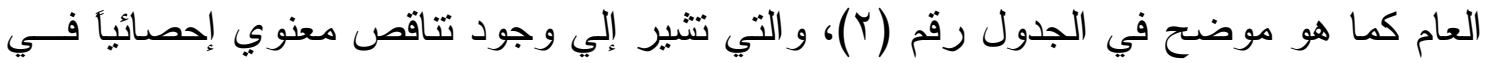

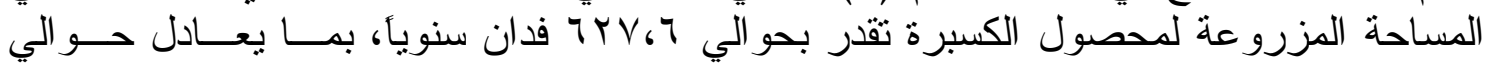

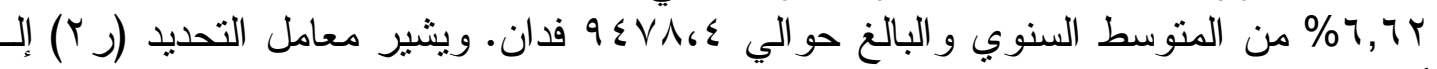

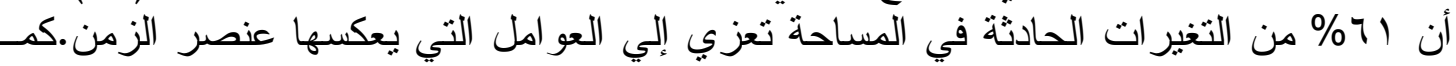

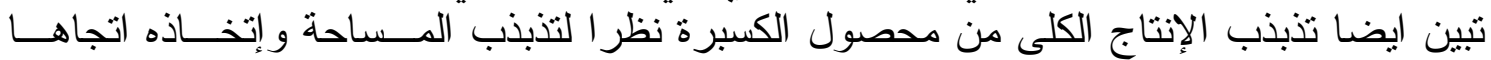

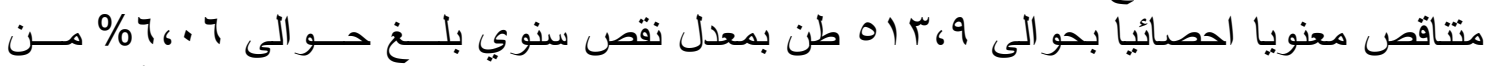

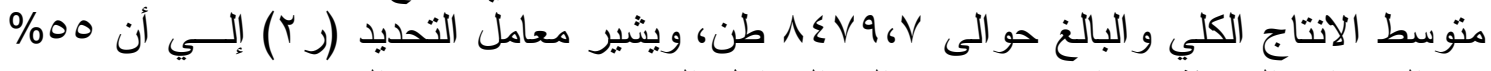

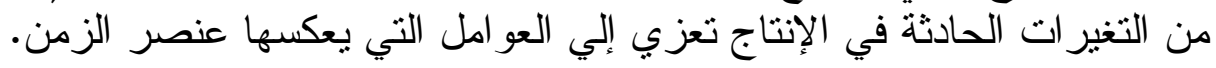

جدول رقم 1 ـ تطور كل من المساحة والإتتاج الكلي لأهم النباتات الطبية والعطرية فى مــصر خــله

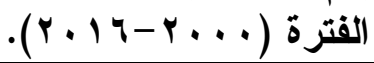

\begin{tabular}{|c|c|c|c|c|c|c|}
\hline \multicolumn{2}{|c|}{ الشمر } & \multicolumn{2}{|c|}{ الكمون } & \multicolumn{2}{|c|}{ الكسبرة } & \multirow[t]{2}{*}{ السنوات } \\
\hline $\begin{array}{l}\text { الإنتاج) } \\
\text { (طن) }\end{array}$ & المساحة & $\begin{array}{l}\text { الإنتاج) } \\
\text { (طن) }\end{array}$ & المساحة & $\begin{array}{l}\text { الإنتاج) } \\
\text { (طن) }\end{array}$ & المساحة & \\
\hline ए人9० & rqVT & Troo & $717 V$ & $9 \leq \ldots$ & $1 \ldots 11$ & $r \ldots$ \\
\hline YVIY & $Y Y \leqslant V$ & . & $V \cdot r q$ & $974 \mathrm{~V}$ & $1 . Y 7 \pi$ & $r \ldots 1$ \\
\hline$r \leqslant r$. & $1 v r q$ & rVAV & $V \wedge I V$ & 1.749 & $1 \leq .7$. & $r \ldots r$ \\
\hline YYOI & $17 r$. & rurv & VTYT & $9 V \cdot V$ & ITVVI & $r \ldots r$ \\
\hline YV.O & $1 \wedge 9 Y$ & rOAO & $\leqslant 910$ & IYYT. & ITVY. & $Y \ldots \varepsilon$ \\
\hline ruq & $r Y \cdot V$ & TVYY & $01 \leqslant r$ & 119977 & $1 \leq \leqslant 00$ & $r \ldots o$ \\
\hline$r \leqslant O V$ & YYYE & $T) \leqslant V$ & TVVY & 1.797 & $11 V V 7$ & $r \ldots T$ \\
\hline ATH & TVMT & rY.A & $\varepsilon I V V$ & $1110 \leqslant$ & $1 \leqslant \varepsilon \leqslant Y$ & $r \ldots V$ \\
\hline rqV & $r 7 \leqslant q$ & ITHA & YIYA & VYqY & $\Lambda \cdot \vee q$ & $r \ldots \lambda$ \\
\hline YTY. & $r \cdot \Lambda$. & 1790 & 1107 & $1.17 \varepsilon$ & 1.194 & $r . . q$ \\
\hline$r V \leqslant \Lambda$ & YIVV & TrT. & $0 \leqslant \leqslant \wedge$ & $111 \mathrm{~V} 0$ & 11998 & $r .1$. \\
\hline TYAV & $r 9.7$ & YIYT & $r \leqslant \wedge q$ & Vos & Q.Yr & $r .11$ \\
\hline 1007 & rqYo & $110 \mathrm{~V}$ & $19 \leq 1$ & $\varepsilon \leqslant \leqslant 0$ & 07.0 & $r \cdot I T$ \\
\hline YOV & $r \cdot \Lambda V$ & IITY & 1Аr & rovt & MTYo & $r .1 T$ \\
\hline$r \cdot V \cdot$ & 1079 & 10.0 & $r \wedge \leq 7$ & 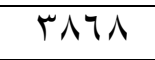 & 1971 & $r \cdot 1 \varepsilon$ \\
\hline $9 \wedge 7$ & 1711 & Y07Y & $19 \leq 4$ & ros. & $r v \cdot q$ & $r .10$ \\
\hline$\varepsilon \leqslant 7 \mu$ & $\Gamma \leq 7 \Gamma$ & 1.11 & IVYV & $r \leqslant r_{0}$ & ro9V & $r .17$ \\
\hline$\Gamma \cdot \wedge \wedge_{6} \wedge$ & $r M \wedge \Lambda_{6} 0$ & $r \nVdash \leqslant V_{6} \wedge$ & $\varepsilon r .969$ & $\wedge \leqslant \vee q_{6} \vee V$ & $9 \leqslant \vee \wedge_{6} \leqslant$ & المتوسط \\
\hline
\end{tabular}


Website: www.aun.edu.eg/faculty_agriculture/journals_issues_form.php E-mail:ajas@aun.edu.eg

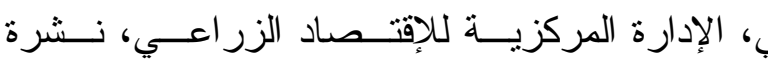

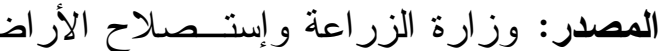
الإحصاءات الزر اعية، أعداد متفرقة.

جدول رقم r. نتائج تقدير الاتجاه الزمني العام لكل من المساحة والاتتاج الكلى لأهم النباتات الطبيــة

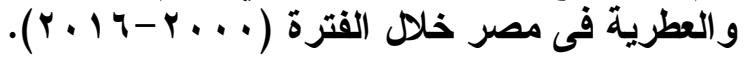

\begin{tabular}{|c|c|c|c|c|c|}
\hline السنوي(\%) & ف & ru & المعادلة & البيان & الححصول \\
\hline צTיד & "Arra & . .71 & 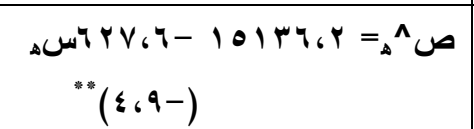 & (فان) & \multirow{2}{*}{ الكسبرة } \\
\hline $9 ., 4$ & $" 1 \Lambda_{6} \cdot r$ & .00 & 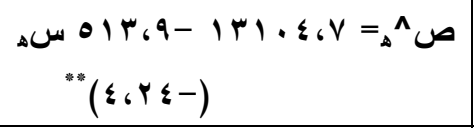 & $\begin{array}{l}\text { الاتناج } \\
\text { (طن) }\end{array}$ & \\
\hline$A_{G} V$ & $" \approx \wedge \wedge, V$ & דיט, & 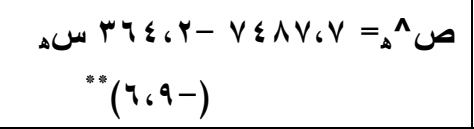 & (فانة) & \multirow{2}{*}{ الكمون } \\
\hline 0,1 & $" 11_{6} 9$ & .099 & 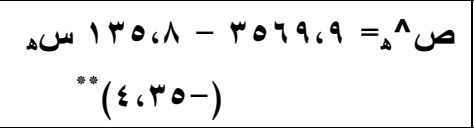 & الاتناج & \\
\hline- & .r) & \& & 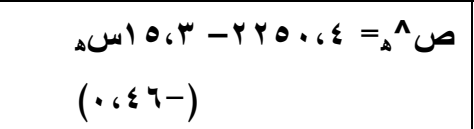 & (فلمان) & \multirow{2}{*}{ الثمر } \\
\hline- & .rT & $\ldots$ & 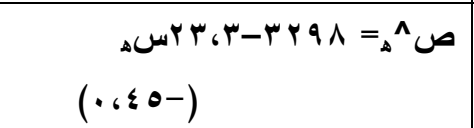 & $\begin{array}{l}\text { الاتتاج } \\
\text { (ط) }\end{array}$ & \\
\hline
\end{tabular}

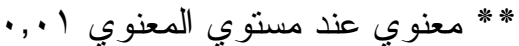

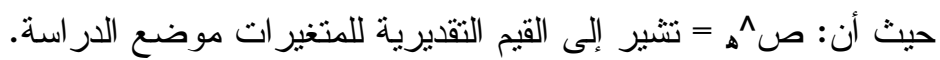

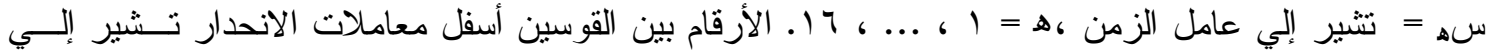
قيمة (ت) المسبوبة. المصدر : حسبت من: جدول رقم (1).

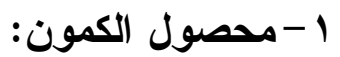

تثنير البيانات الواردة في الجدول رقم (1)، إلي أن المساحة المزروعـــة مــن محسـصول

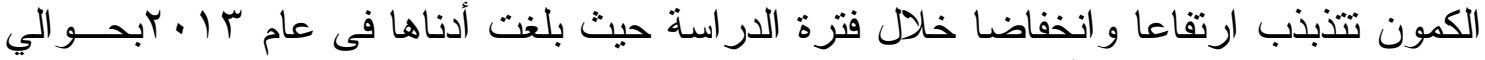

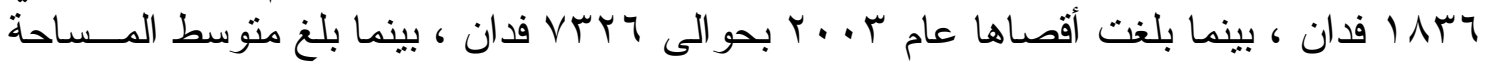

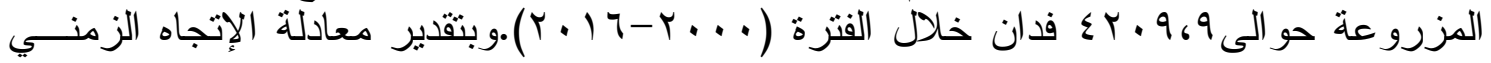

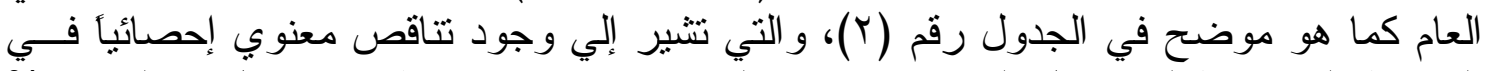

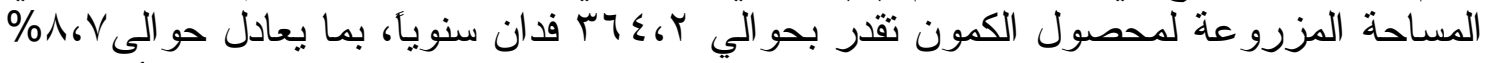

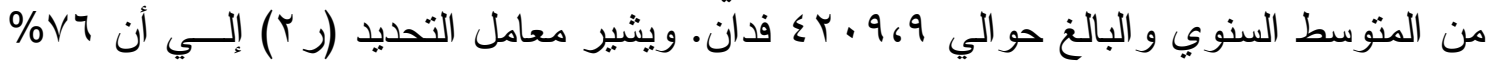

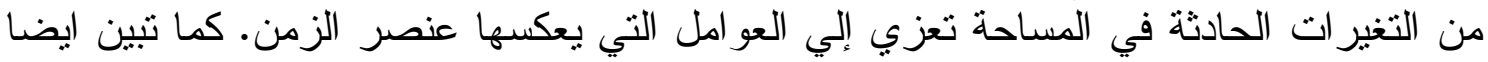

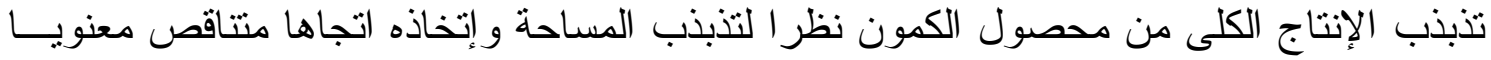

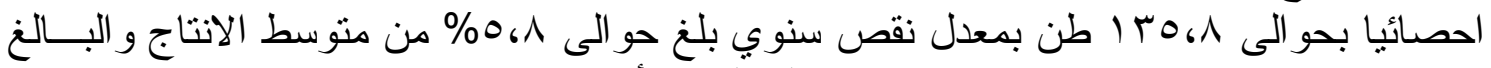

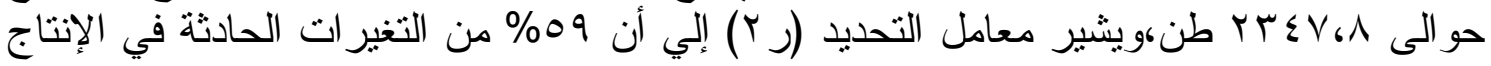
تعزي إلي العوامل التي يعكسها عنصر الزمن. 


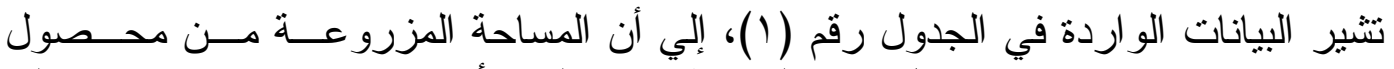

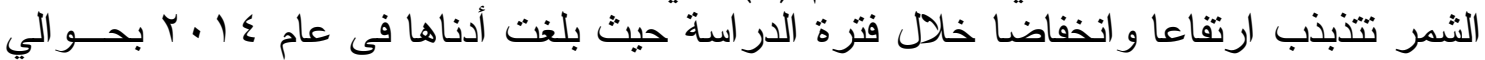

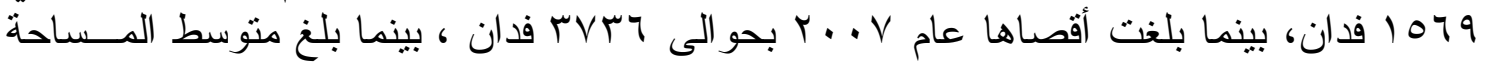

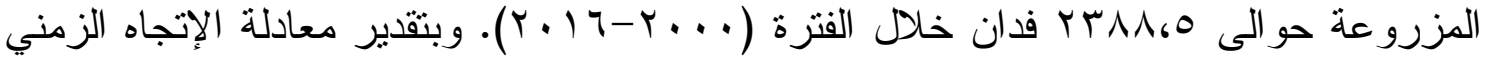

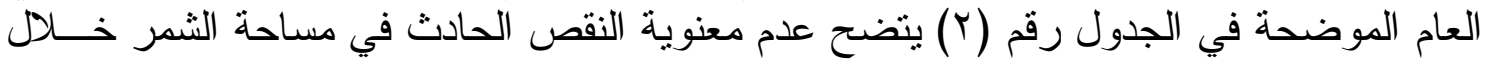

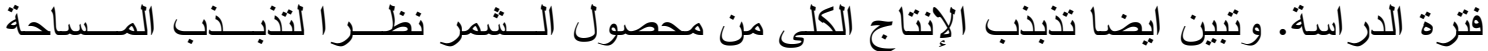

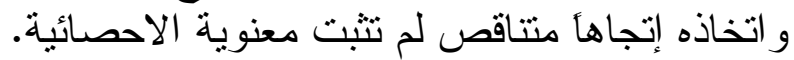

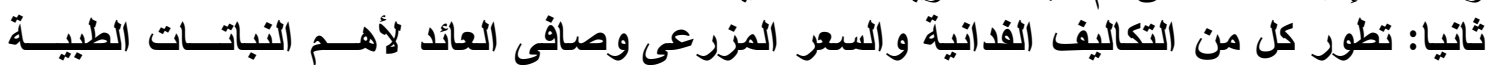
و العطرية فى مصر خلا: فترة فئرة الدراسة: 1 - 2 - محصول الكسبرة:

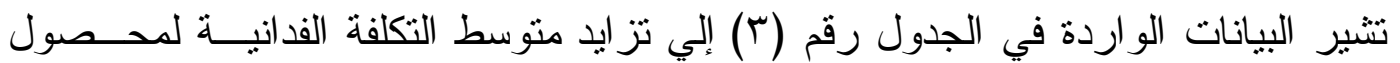

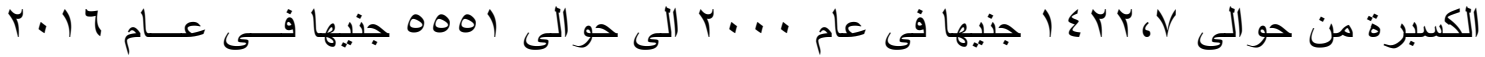

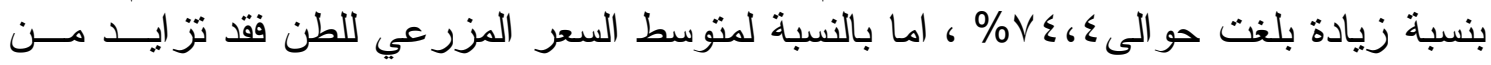

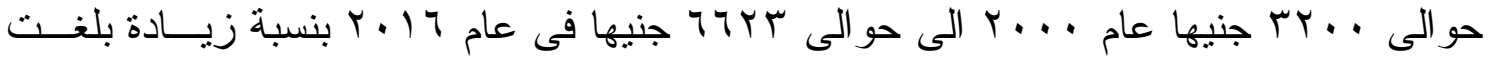

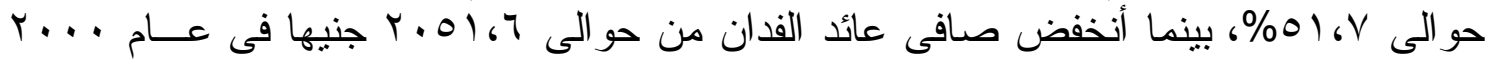

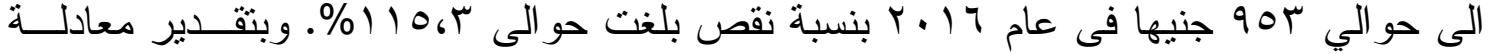

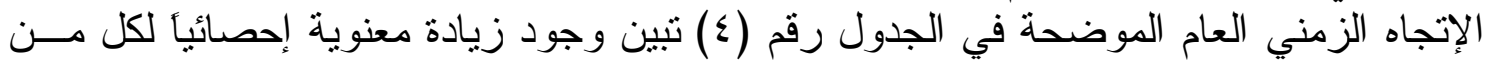

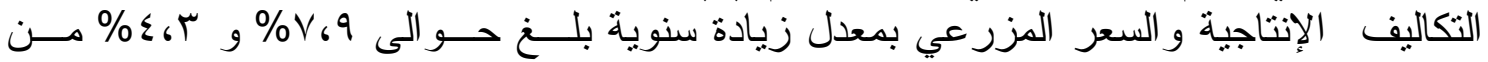

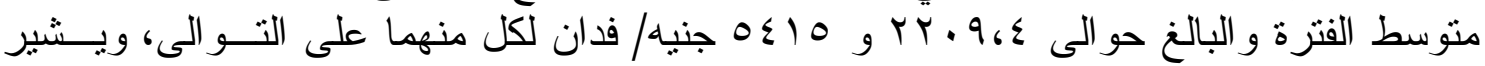

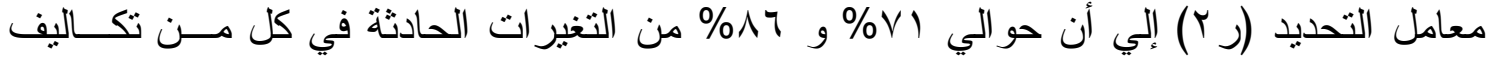

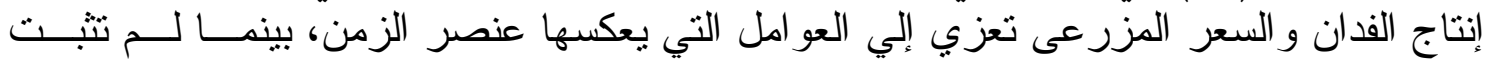
معنوية الزيادة الحادث في صافى عائد الفدان خلال فترة الدر اسلة.

جدول رقم ب. تطور كل من التكاليف الفدانية والسعر المزرعى وصافى العائد لأهم النباتــات الطبيـة

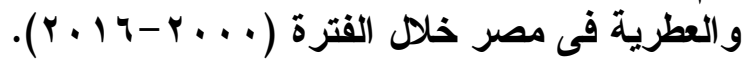

\begin{tabular}{|c|c|c|c|c|c|c|c|c|c|}
\hline \multicolumn{3}{|c|}{ الشمر } & \multicolumn{3}{|c|}{ الكمون } & \multicolumn{3}{|c|}{ الكسبرة } & \multirow{2}{*}{ المحصول } \\
\hline (جنيه/ (ائد) & (جنيه/طن) & (الفالنية & (جنيهم/ فل) & جنيه/طن السعي & (القاليف) & (جنيه/ فائدى) & (جنيه/طن) & الفاليف & \\
\hline$\varepsilon Y \wedge \varepsilon$ & «ov. & IV.Y.E & r779 & $90 \ldots$ & $r \ldots \varepsilon_{6} q$ & $r .0167$ & $r v^{\prime}$. & $1 \leqslant Y_{6} V$ & $r \ldots$ \\
\hline TOYA & $\varepsilon r \ldots$ & $17 r \varepsilon_{6} Y$ & ro 09 & $99 \ldots$ & 19906 & TrO1.9 & rA.. & $1 \pi \mid \varepsilon_{0}$ & $r \ldots l$ \\
\hline$\varepsilon Y Y q$ & $\sum 1 \ldots$ & 101.67 & Yq9ई & $1,1 \ldots$ & $1 V_{9} V_{6} 0$ & 101960 & r... & $1 Y V V_{6} V$ & $r \ldots r$ \\
\hline$\varepsilon . M T$ & $\varepsilon \ldots$ & $10 Y \varepsilon_{6}$ & $r \leqslant q r$ & $1 . \wedge \ldots$ & $1 \wedge 7 \varepsilon_{6}$ & 1789 & $r q \ldots$ & $1 Y \wedge 0_{6}$. & $r \ldots r$ \\
\hline$\varepsilon \vee 0 q$ & $\varepsilon \leqslant 0$. & 17.066 & rq & $90 \ldots$ & 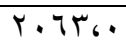 & rIVA & $\varepsilon \ldots$ & $1 \% \wedge T_{6}$ & $r_{\ldots} \ldots \varepsilon$ \\
\hline$\varepsilon 0 \wedge 7$ & $\varepsilon \cdot r V$ & $|\wedge|$. & $r \ldots \tau$ & $90 \%$. & $r . r \sigma_{6}$, & KMA.GT & \&YO. & $1 \leqslant r_{6}$. & $r \ldots o$ \\
\hline EOTY & $\varepsilon / Y q$ & 19.96. & rrIT & $97 \varepsilon$. & TYAYG。 & YITY & $\varepsilon \varepsilon, 1$ & $1 \wedge r q_{6}$. & $r \ldots r$ \\
\hline$\varepsilon r \uparrow V$ & $\varepsilon r \leqslant \varepsilon$ & $1 \wedge \Sigma 16$ & OrVo & IYKVO & $r r \leqslant V_{6}$. & ro90 & $7 \ldots Y$ & 11916 & $r \ldots v$ \\
\hline orß & 7.19 & $Y_{.} T_{T_{6}}$ & TIYY & 101.0 & r779 & roqr & $\pi r .0$ & $r \cdot T \varepsilon_{6}$ & $r \ldots \lambda$ \\
\hline $0 \leqslant 11$ & $7 / Y 7$ & TYVI6. & $7.9 \mathrm{~V}$ & 10490 & זדז4. & $\varepsilon \cdot \mu r$ & $7 r .0$ & $r Y_{0} \varepsilon_{6}$ & $r \ldots q$ \\
\hline r90 & 7rYq & $r \leqslant 0 r_{6}$. & TrVA & $10 \leqslant 97$ & $r \wedge \leqslant r_{6} \cdot$ & $\varepsilon \cdot \Gamma \xi$ & $7 \varepsilon .1$ & TrוT, & $r .1$. \\
\hline$\varepsilon \varepsilon r Y$ & THAY & TVAT,。 & $V \cdot T$ & ITRTY & rqr.6. & $r . \leqslant q$ & 70.1 & rTqY.。 & $r \cdot 11$ \\
\hline$\{7 Y$ & $7 \leqslant r \wedge$ & 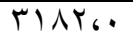 & $71) \varepsilon$ & $17 \leqslant \Lambda$. & $r \cdot \leqslant 160$ & rVAO & $70 \mathrm{VI}$ & $r \leqslant 0 r_{6}$. & $r \cdot I r$ \\
\hline$\varepsilon \wedge \leqslant 0$ & roro & Tा & TYM & TITYV & TYMI & $r \wedge 90$ & $70 \wedge Y$ & TYAV & $r \cdot 1 r$ \\
\hline OYMT & $70 \mathrm{~V}$. & $r \leqslant r$. & 0179 & $177 \leqslant 9$ & r700 & एकाוा & 77.5 & rqYo & $r \cdot I \varepsilon$ \\
\hline$\varepsilon \leqslant V)$ & אזTY & אזדצr & $7 \varepsilon \cdot 1$ & 1778. & rarv & דודיז & TIIY & rlor & $r .10$ \\
\hline
\end{tabular}


Website: www.aun.edu.eg/faculty_agriculture/journals_issues_form.php E-mail:ajas@aun.edu.eg

\begin{tabular}{|c|c|c|c|c|c|c|c|c|c|}
\hline r90V & $7 V T 1$ & OVYT & $\varepsilon V V Y$ & IVTKE & $711 \varepsilon$ & 904 & TITr & 0001 & $r .17$ \\
\hline$\varepsilon \leqslant \leqslant 7 ، \leqslant$ & Or.0.0 & $r \leqslant 9 r_{6} 9$ & $\sum \tau, \Gamma_{6} \wedge$ & ITHV & rV91،V & YVVY,T & $0 \leqslant 10$ & KY.96 & المتوسط \\
\hline
\end{tabular}

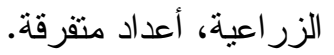

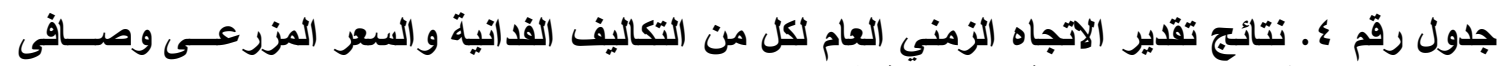

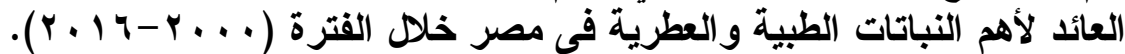

\begin{tabular}{|c|c|c|c|c|c|}
\hline السنوي (التغير) & ف & ru & المعادلة & البيان & المحصول \\
\hline$V_{6} 9$ & 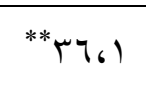 & . 61 & 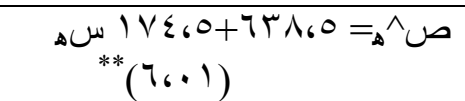 & تكاليف الانتاج) & \multirow{3}{*}{ الكسبرة } \\
\hline \&, & ${ }^{* * *} \wedge q_{6} . \varepsilon$ & . & 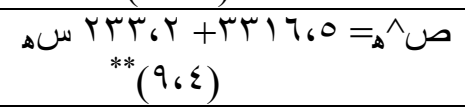 & السعر المزرعي عي (جني) & \\
\hline - & r. 9 & .617 & 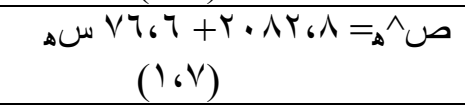 & (جنيه/ فدان) العائد & \\
\hline $7 ، \varepsilon$ & $\left.{ }^{* *}+V_{6}\right)$ & .61 & 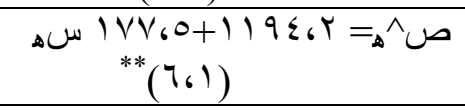 & تكاليف الانتاج) & \multirow{3}{*}{ الكمون } \\
\hline r.9 & 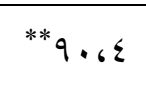 & . 617 & 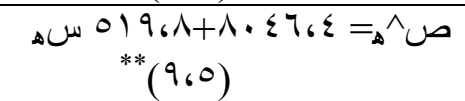 & السعر المزرعي) & \\
\hline - & ... & $\cdots$ & 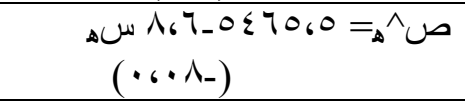 & 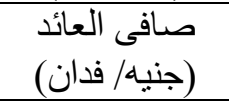 & \\
\hline$V_{6} 7$ & 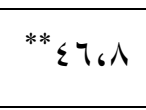 & .674 & 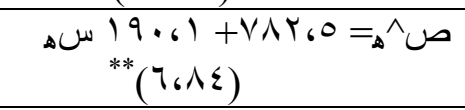 & تكاليف الانتاج & \multirow{3}{*}{ الثمر } \\
\hline r.T & ${ }^{* *} \mid 0,11$ & .0. & 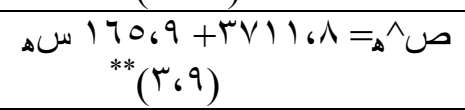 & السعز المزرعي) & \\
\hline - & .610 & .61 & 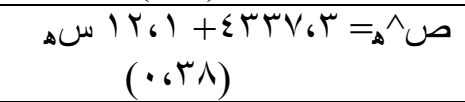 & 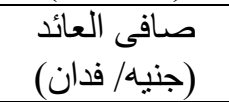 & \\
\hline
\end{tabular}

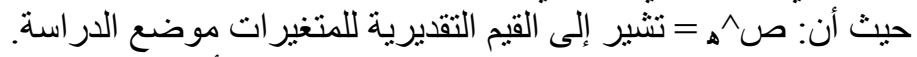

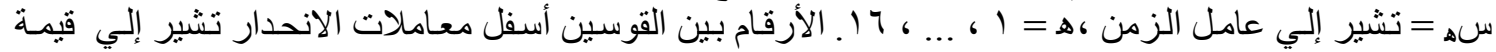

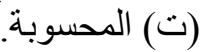

$$
\text { المصدر: حسبت من: جدول رقم (r). }
$$

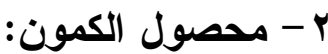

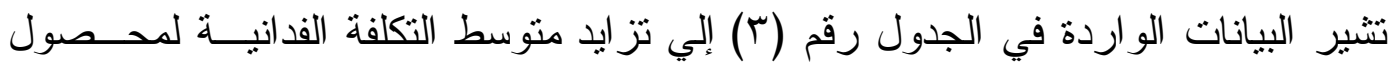

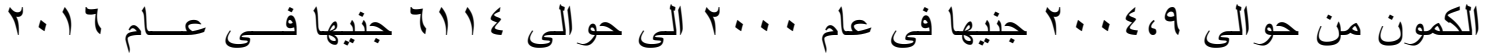

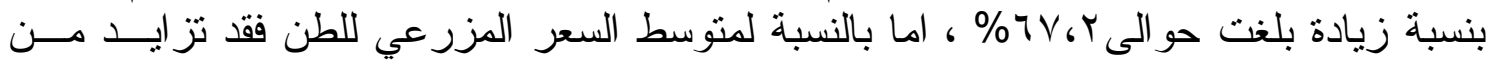

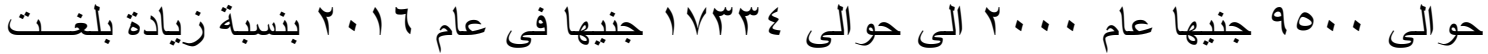

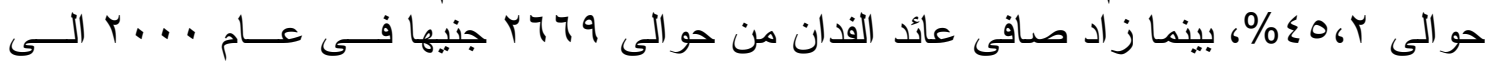

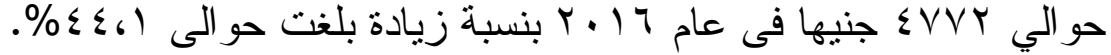

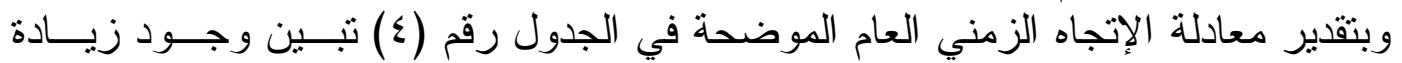

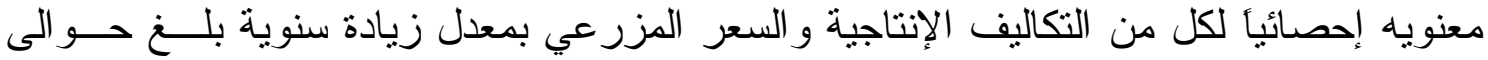

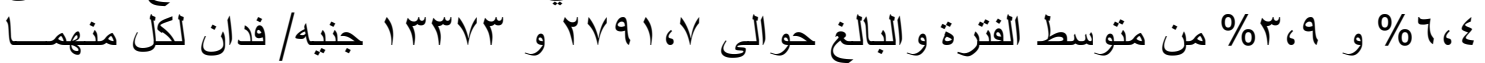

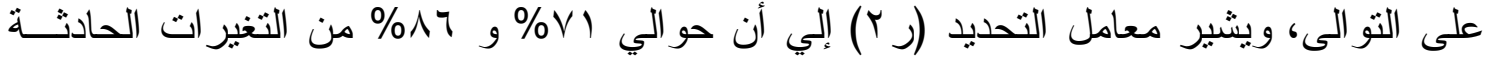




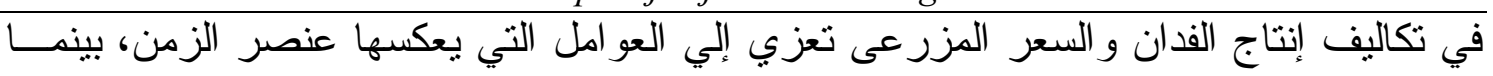

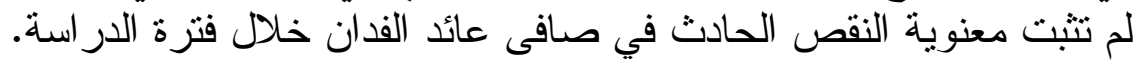

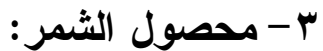

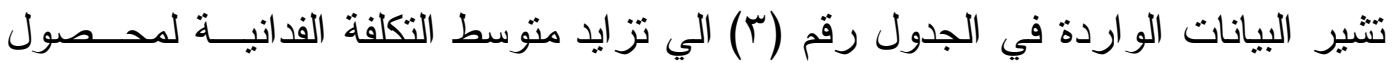

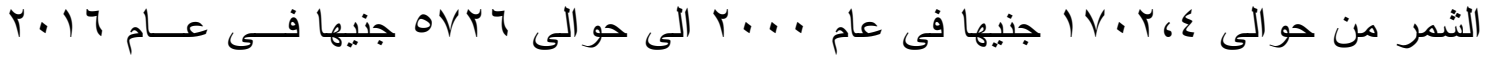

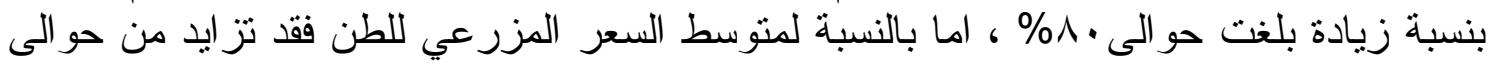

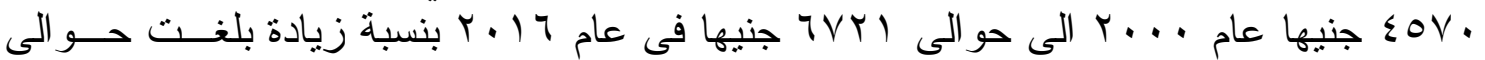

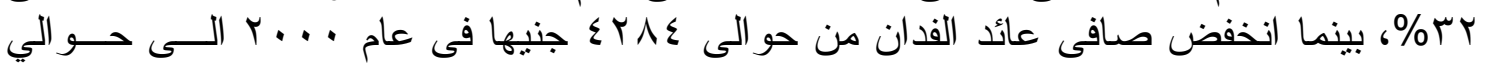

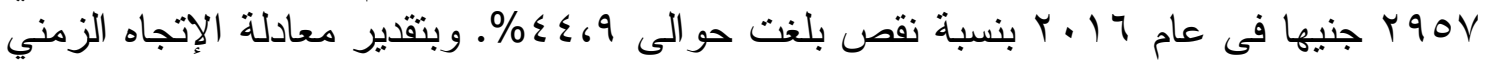

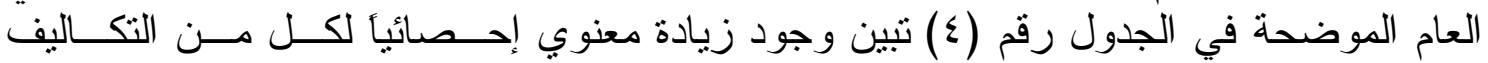

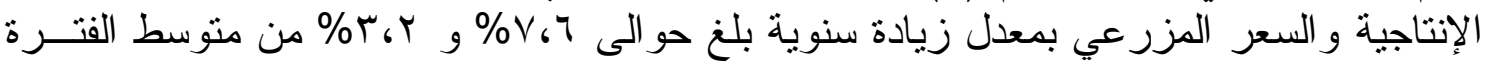

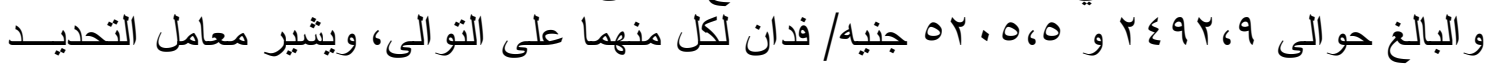

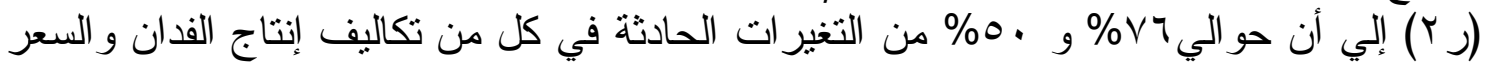

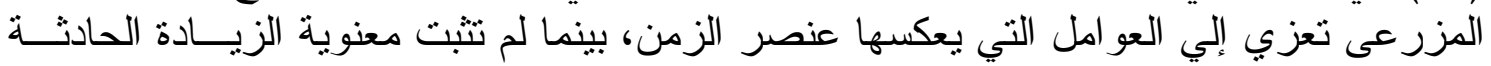

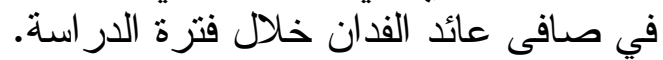
ثالثا: التجارة الخارجية لأهم النباتات الطبية والعطرية فئة في مصر خلا فترة الدراسة: 1- الطور الصادرات المصرية:

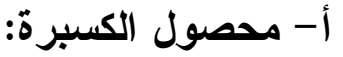

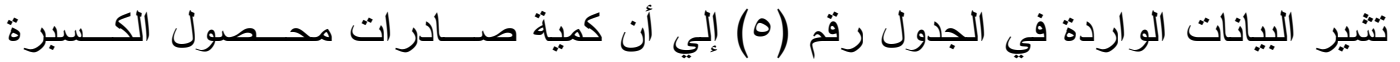

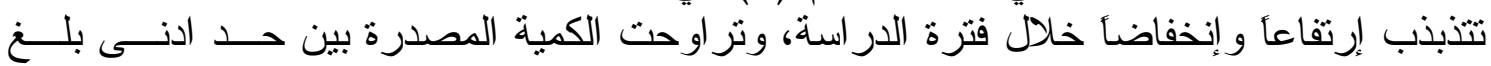

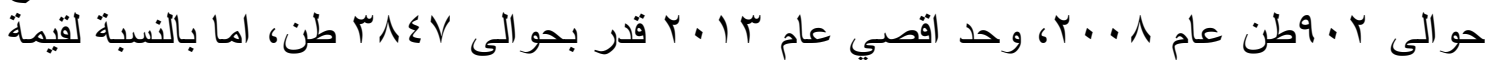

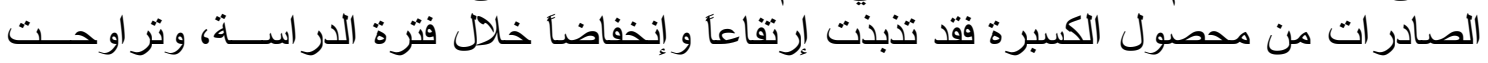

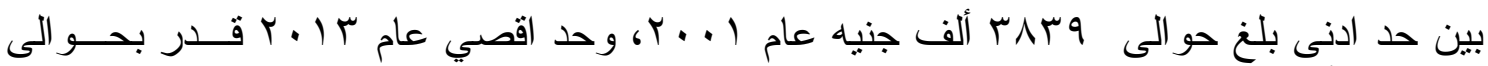
الر roVA

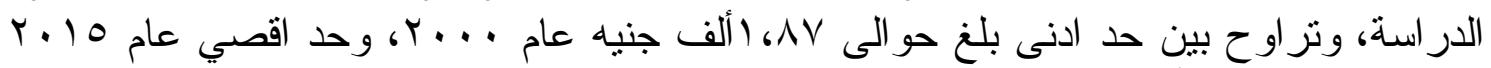

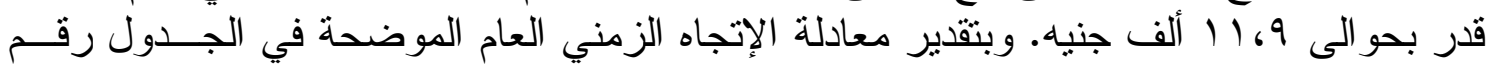

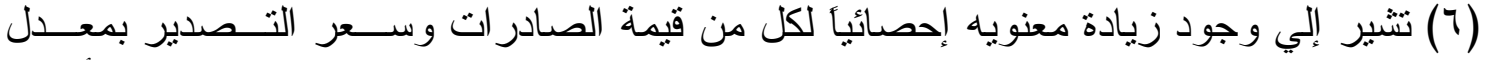

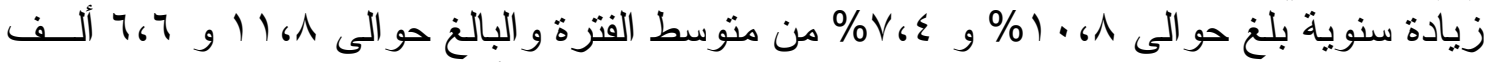

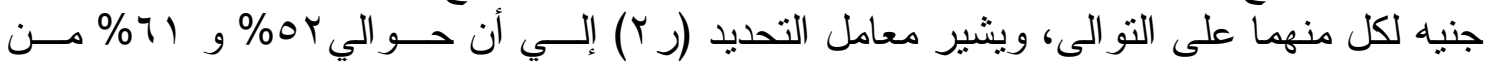

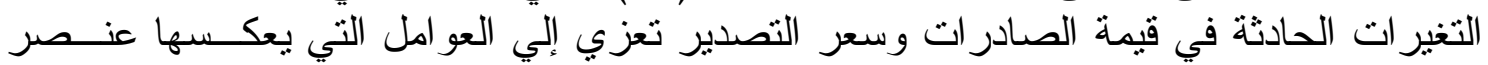
الزمن، بينما لم تثتبت معنوية الزيادة الحادث في كمية الصية الصادر ات خلادل فترة الدر اسة.

ب- محصول الكمون:

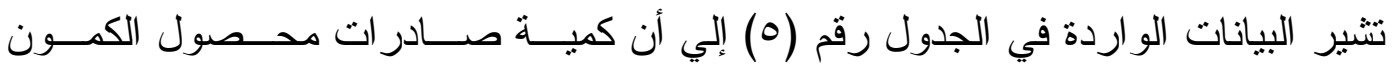

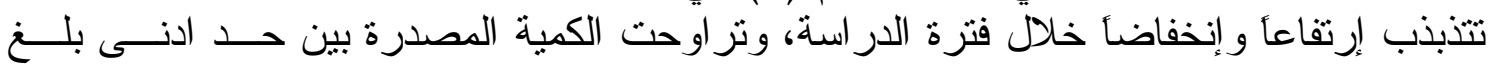

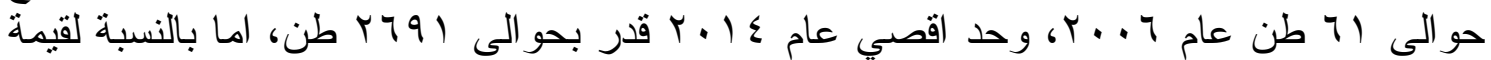

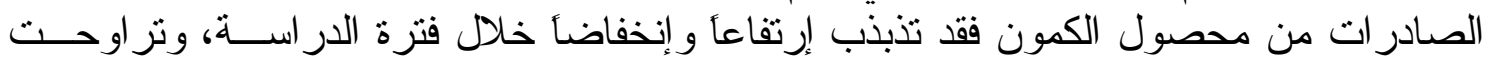

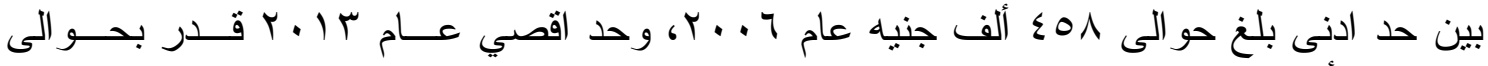

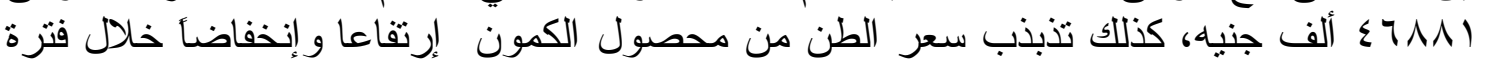

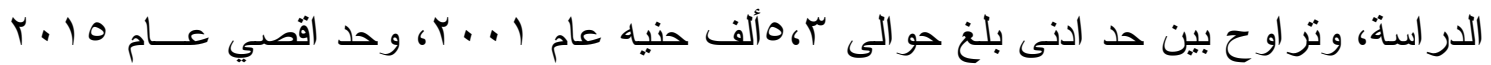

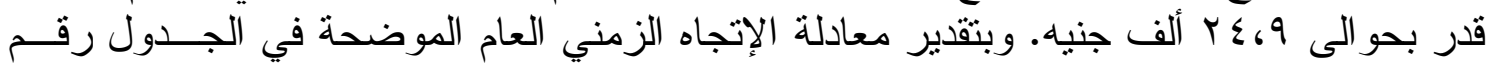

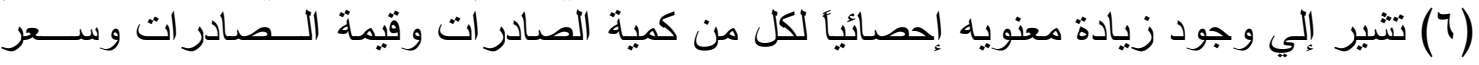


Website: www.aun.edu.eg/faculty_agriculture/journals_issues_form.php E-mail:ajas@aun.edu.eg

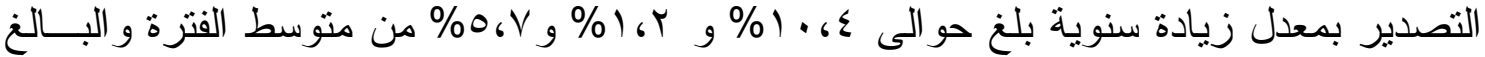

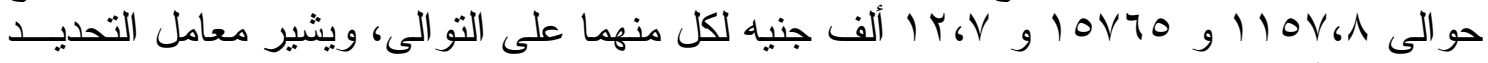

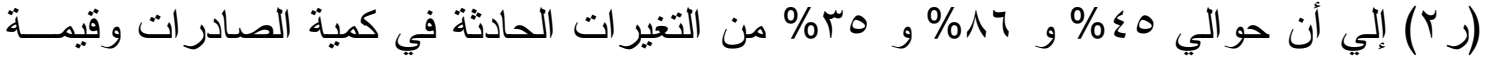

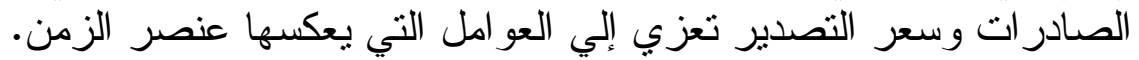

جدول رقم ه. تطور الصادرات المصرية لأهم النباتات الطبية والعطريــة فـى مـصر خــلا الفتـرة

\begin{tabular}{|c|c|c|c|c|c|c|c|c|c|}
\hline \multicolumn{3}{|c|}{ الشمر } & \multicolumn{3}{|c|}{ الكمون } & \multicolumn{3}{|c|}{ الكسبرة } & \multirow[t]{2}{*}{ |لمحصول } \\
\hline جنيه/ طن) (السعر (ألف & (بالف جنيه) & (طن) & (جنيه/ طن) ألف) & (بالف جنيهة / القيمة & (طنية/ & جنيه/ ألفعر طن & جنيمة / (بالف & (طن) الكمية/ & \\
\hline$r_{60}$ & $1 \ldots \leq 7,0$ & एq१V & $\varepsilon 69$ & $\varepsilon 0 \wedge 1$ & $9 \leq 1$ & $1, \Delta V$ & 0917 & 1199 & $r \ldots$ \\
\hline$T_{6} \varepsilon$ & $V \vee I$ & TYOO & 0,1 & LYTK & 199 & T.17 & rNHq & $|\vee \wedge|$ & $r \ldots 1$ \\
\hline T.9 & 9707 & TYVV & $\Lambda_{6} \varepsilon$ & एาT & $\varepsilon r r$ & $r_{6} \varepsilon r$ & $0 . \Sigma T$ & r. Vo & $r \ldots r$ \\
\hline$\varepsilon_{6} V$ & ITY. & $r \leqslant Y$. & 1.61 & $\varepsilon Y \wedge l$ & $\varepsilon Y \xi$ & E.rq & รOH & 1.01 & $r \ldots r$ \\
\hline 0,5 & $r r \leq 0 \wedge$ & \&MTV & 1.61 & $r \leqslant Y V$ & $\Gamma \varepsilon$. & $7.7 \varepsilon$ & $7 \varepsilon .0$ & $97 \varepsilon$ & $r \ldots \varepsilon$ \\
\hline$\varepsilon .9$ & $19 \wedge \wedge$. & $\varepsilon .9 V$ & 9.7 & Y)^9 & YYA & $\varepsilon_{6} \wedge t$ & $\sum \wedge \wedge q$ & $1 \cdots v$ & $Y . .0$ \\
\hline 0.5 & rToqr & 7419 & $V_{6} 0$ & $\leqslant 0 \wedge$ & 71 & $\varepsilon, Y \varepsilon$ & 0.199 & 1191 & $Y . . T$ \\
\hline 0.0 & rรVะ. & TIYI & 1.61 & TNIT & Tol & $\varepsilon_{6 \Delta \Lambda}$ & NOY & IVTr & $r . v$ \\
\hline 9.9 & एะVะ. & roo. & 1964 & TlOVT & 1150 & $96 \leqslant 0$ & NOY & $9 \cdot r$ & $r \ldots \Lambda$ \\
\hline$\varepsilon_{6} \wedge$ & YTYA. & $0 \leqslant 70$ & 1062 & MVA. & Y.01 & 0,17 & AYIV.q & $109 \varepsilon$ & $r \ldots q$ \\
\hline $0, T$ & 7.7 .7 & $1119 \varepsilon$ & $1 \Lambda_{6} \cdot \varepsilon$ & YIT & I11 & $1.67 \varepsilon$ & $177006 \mathrm{~V}$ & 1070 & $r+1$. \\
\hline $116 \%$ & $9 \vee V 17$ & $\wedge V \cdot 9$ & $1 V_{6} 0$ & КАтル. & 17ro & 11.01 & $10 \leqslant \wedge r$ & דוTו & $Y .11$ \\
\hline 196.0 & 10110 & OVTV & 196.5 & 19790 & $r \cdot \Lambda \varepsilon$ & $V_{6} \wedge \varepsilon$ & ITYY & YIT. & $r \cdot 1 r$ \\
\hline 1061 & $\varepsilon \wedge 0 \wedge 9$ & TrI. & $r \cdot . \wedge$ & $\left.\sum \curlyvee \wedge \wedge\right)$ & TYOT & $1 \cdot 6 \cdot r$ & rAOVA & $r \wedge \leqslant V$ & $r .1 r$ \\
\hline 961 & AVY. & 971 & $\left.V_{6}\right)$ & 119990 & 1791 & 7.9. & 1 1) & $r \ldots q$ & $r+1 \leq$ \\
\hline 1.64 & 1071 & $V \leqslant 0$ & $r \leq 69$ & $1.7 \times 7$ & $\varepsilon r q$ & 1169 & $r \mu$ & 1945 & $r .10$ \\
\hline $116 r$ & Y0.T & YYT & $\Lambda_{6} Y$ & rIAro & $r \neg \leqslant \Lambda$ & $\mathrm{V} .9$ & IETIV & 1117 & $r .17$ \\
\hline$V_{6} 7$ & דוT" & $\varepsilon \leqslant 99.7$ & $1 r_{6} \mathrm{~V}$ & $10 V 70$ & $110 V_{6} \mathrm{~A}$ & 7.7 & $11 \mathrm{Vrq}$ & $1 V V \varepsilon$ & المتوسط \\
\hline
\end{tabular}

المصدر: الجهاز المركزي للتعبئة العامة و الإحصاء، نشرة التجارة الخارجية، أعداد متفرقة.

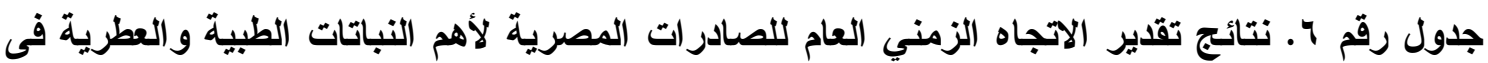

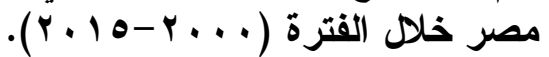

\begin{tabular}{|c|c|c|c|c|c|}
\hline 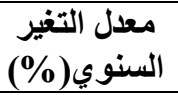 & ف & ז & المعادلة & البيان & المحصول \\
\hline 2. & . $6 \mathrm{TV}$ & $\cdot 6 \cdot r$ & 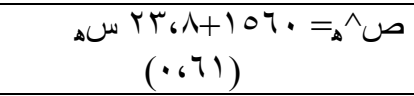 & كمية الصادر ات & \multirow{3}{*}{ الكسبرة } \\
\hline $1 \cdot 61$ & ** 17 ، & or & 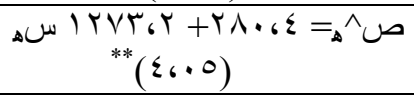 & قيمة الصادر ات & \\
\hline$V_{6} \varepsilon$ & ${ }^{* *} Y_{Y} Y_{6}$ & .671 & 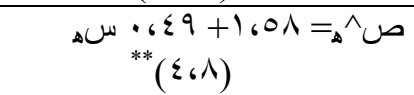 & 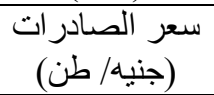 & \\
\hline $1 \cdot 6 \varepsilon$ & ** I Y 6 r & $.6 \leqslant 0$ & 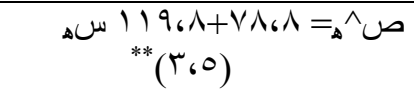 & كمية الصادر ات & \multirow{3}{*}{ الكمون } \\
\hline $16 r$ & **a. ، & . 6А7 & 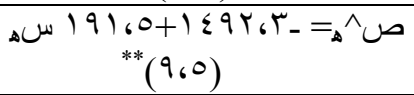 & قيمة الصادر ات & \\
\hline \multirow[t]{2}{*}{$0 . V$} & $\left.{ }^{*} \Lambda_{6}\right)$ & ס 6 . & 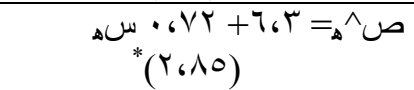 & $\begin{array}{l}\text { سعر الصنادر ات طن) } \\
\text { (جنيه/ }\end{array}$ & \\
\hline & . . &...$r$ & $.679-01776 v=1$ & كمية الصلاربات & الشمر \\
\hline
\end{tabular}


http://ajas.journals.ekb.eg/

\begin{tabular}{|c|c|c|c|c|}
\hline & & & $\left(\cdot\left(0 \Lambda_{-}\right)\right.$ & (طن) \\
\hline - & 1,5 & $\cdot 6 \mathrm{~V}$ & 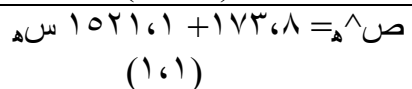 & قيمة الصنادرات \\
\hline 961 & ** Y & .01 & 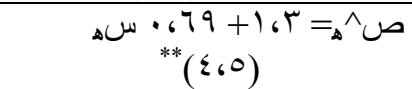 & ستر الصنادرات طنيه) \\
\hline
\end{tabular}

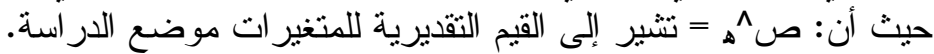

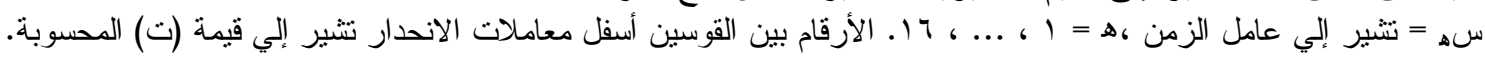

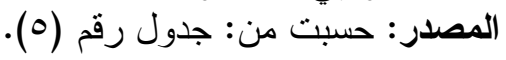

\section{ج- محصول الشمر:}

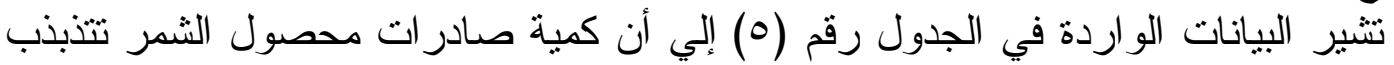

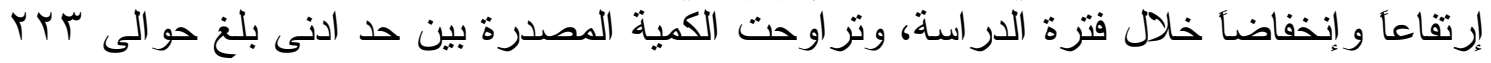

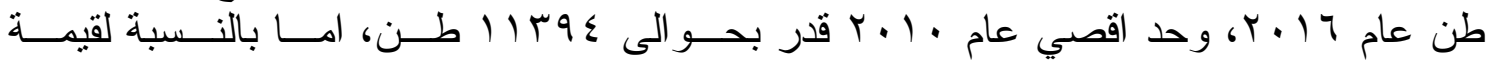

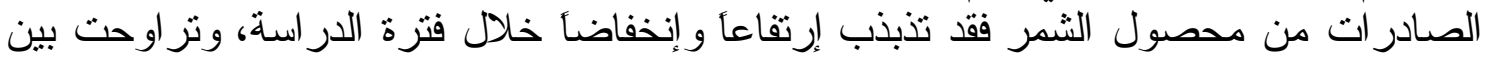

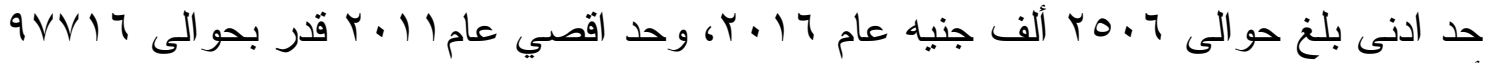

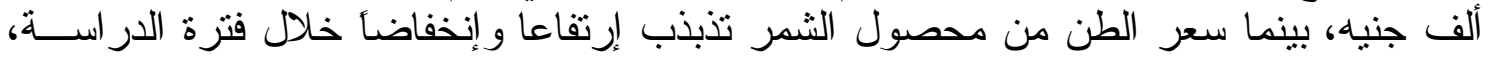

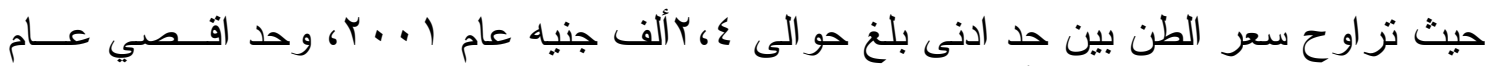

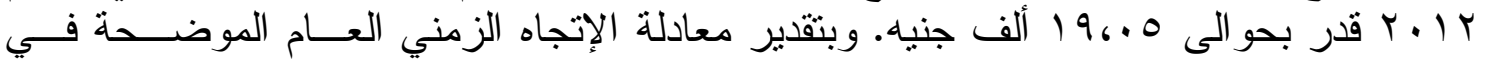

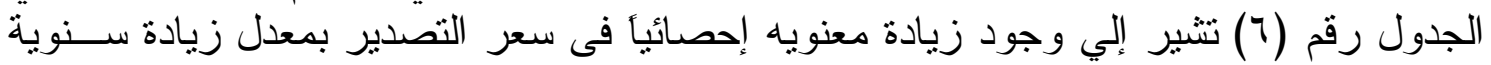

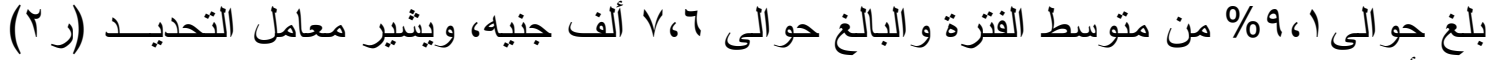

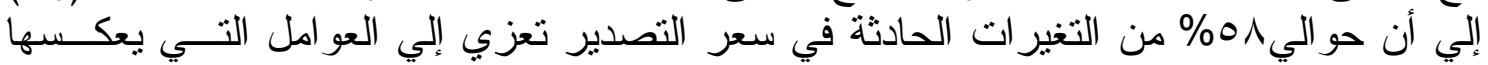
عنصر الزمن، بينما لم نثبت معنوية الزيادة الحادث في كمية الصادر ات وقيمة الصادر ات خلادل فتزة الدر اسة. ب - التوزيع الجغر افي للصادرات المصرية من أهم النباتات الطبـــة والعطريــة خــلال فتـرة أ- أس: محصول الكسبرة:

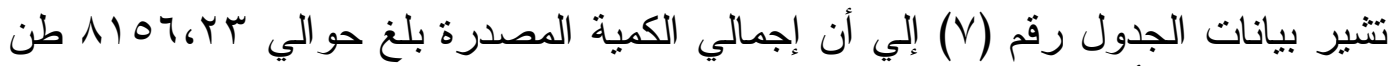

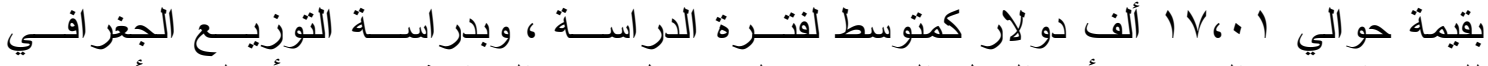

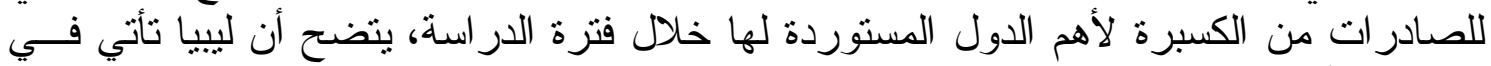

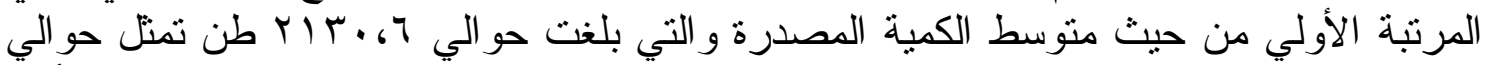

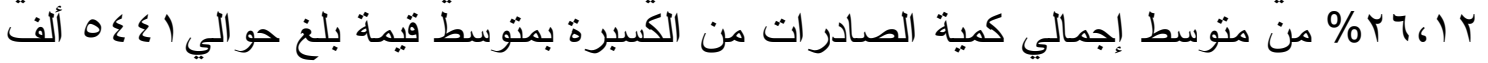

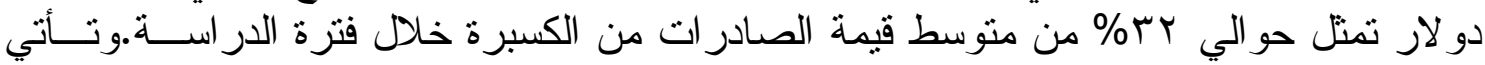

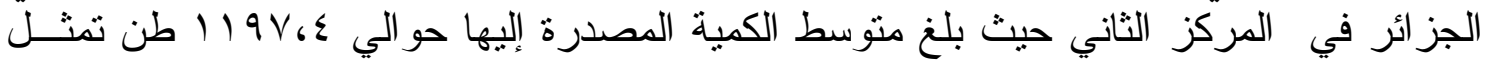

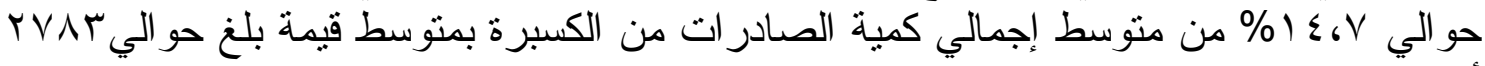

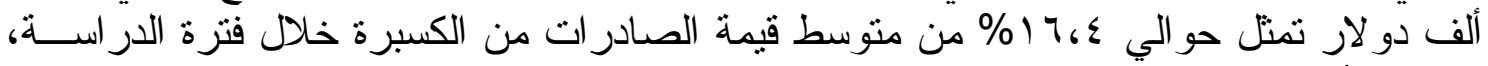

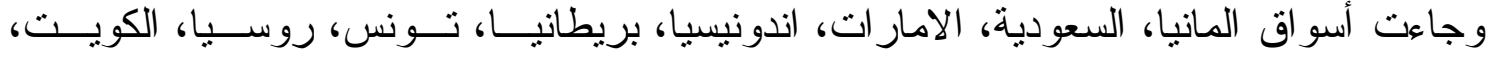

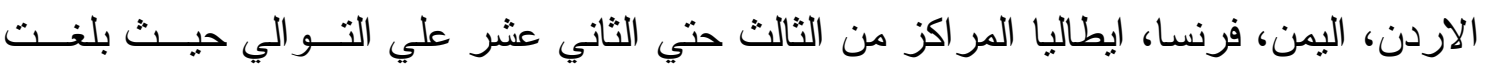

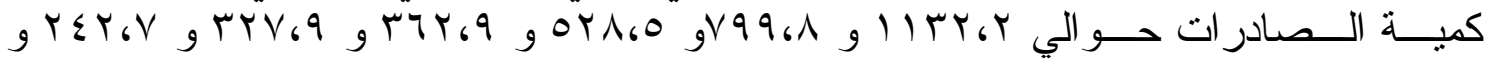
I

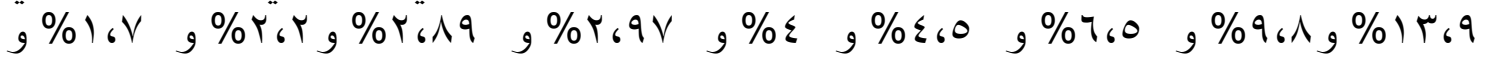

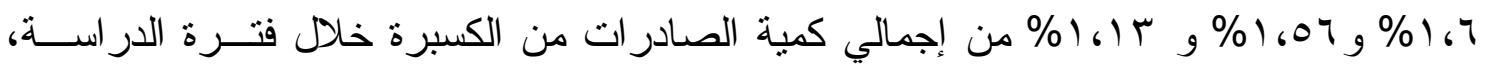

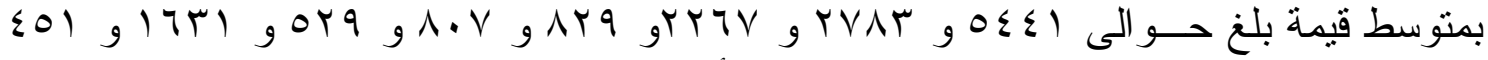

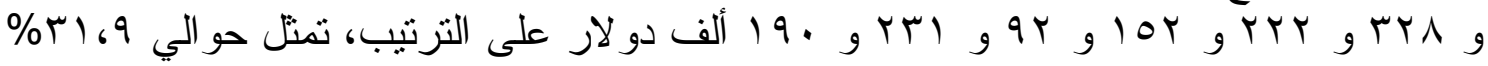




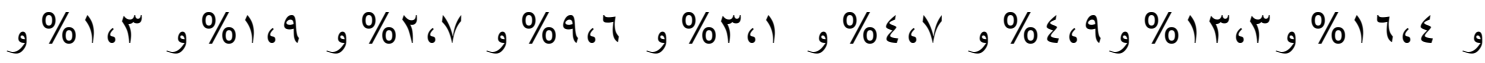

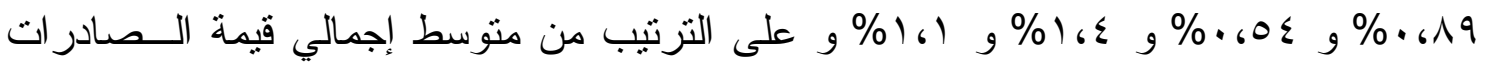

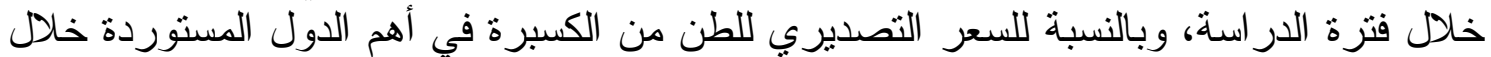

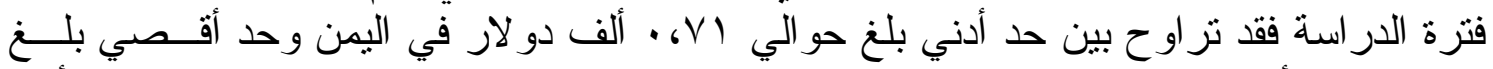

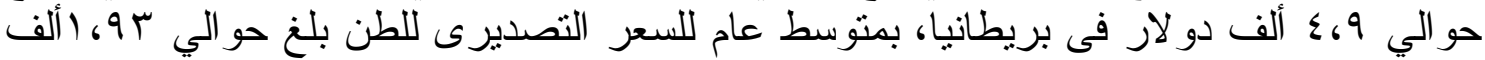

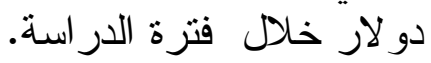


جدول رقم V. التوزيع الجغرافي للصادرات من محصول الكسبرة إلي أهم الدول المستوردة له خــله

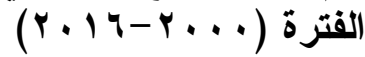

\begin{tabular}{|c|c|c|c|c|c|}
\hline (ألف دولار) & $\%$ & (ألف دولار) & $\%$ & الكمية & الاولة \\
\hline$r_{6} 00$ & 11.99 & $0 \leq \leqslant 1$ & Y7. IT & rוT. & ليبيا \\
\hline r.MT & 17674 & rVAT & $1 \leq 671$ & $119 \mathrm{~V} 6 \mathrm{VV}$ & الجزائر \\
\hline r & سז، & rrTV & $\mu_{6} \wedge \Lambda$ & $\left\|T r_{6}\right\| V$ & المانيا \\
\hline $16 \cdot \varepsilon$ & $\varepsilon_{6} \wedge V$ & $\wedge r q$ & 9.11 & $\vee 9 q_{6} \wedge r$ & السعودية \\
\hline $1.0 Y$ & $\varepsilon_{6} V \varepsilon$ & $\Lambda \cdot V$ & $7, \leqslant 1$ & $O Y \wedge_{6} 0 \leqslant$ & الامـارات \\
\hline $16 \leqslant 0$ & r.11 & org & 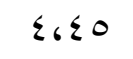 & r r & اندونيسيا \\
\hline$\varepsilon .9 V$ & 9.09 & 1741 & $\varepsilon_{6} \cdot r$ & TrV 6 Ao & بريطانيا \\
\hline 1,10 & T.70 & $\leqslant 01$ & r.9V & $r \leq r_{6} V T$ & تونس \\
\hline 1.41 & 1.94 & rrA & Y.八9 & $r+76.9$ & روسيا \\
\hline I.TY & 1,41 & TrY & $r_{6} T_{0}$ & 189689 & الكويت \\
\hline 16.9 & .619 & $10 r$ & $16 \%$ & $1 \% \Lambda_{6} 97$ & الإردن \\
\hline $.6 \times 1$ & $.60 \leqslant$ & 94 & 1.01 & $1 Y \wedge .97$ & اليمن \\
\hline 1.11 & 1.50 & YMI & 1.07 & $1 Y V_{6} \leq q$ & فرنسا \\
\hline$r_{6} .0$ & 1.14 & 19. & 1.14 & $9 Y_{6} 0 r^{\prime}$ & ايطاليا \\
\hline$r_{6} \cdot 1$ & 7.19 & 1.04 & 7.0. & ro. ، $\leqslant 7$ & إخري \\
\hline 1.94 & $1 \ldots$ & $1 V \ldots 7$ & $1 \ldots$ & N107,rr & الجملة \\
\hline
\end{tabular}

المصدر: وزارة الصناعة و التجارة الخارجية، الهيئة العامة للرقابة علي الصادرات و الواردات، بيانات غير منشورة.

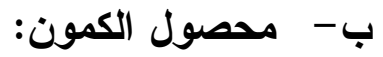

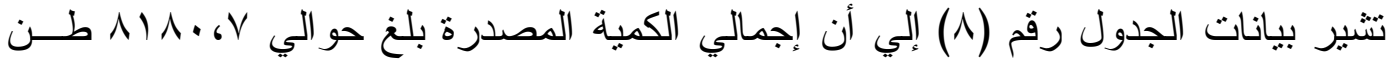

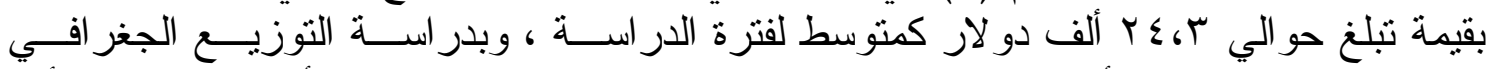

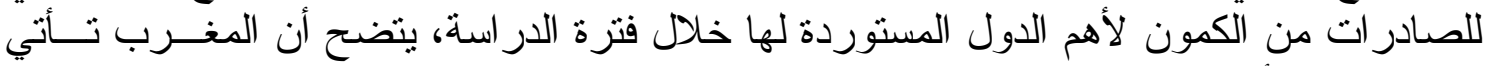

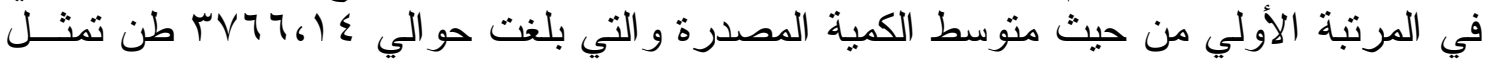

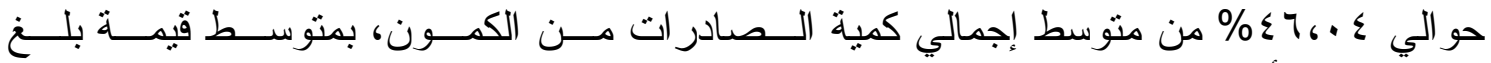

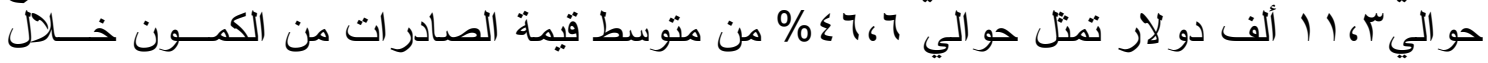

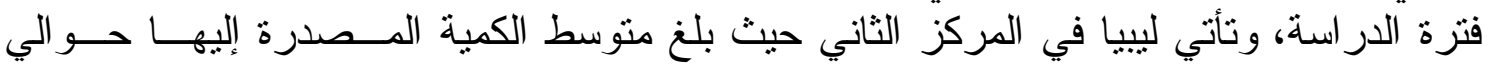

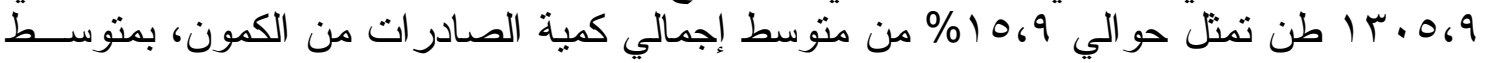

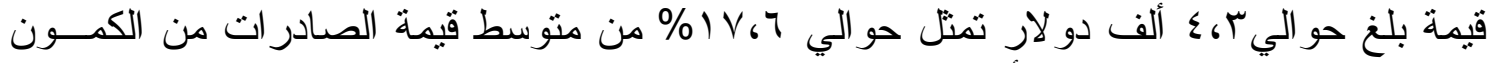

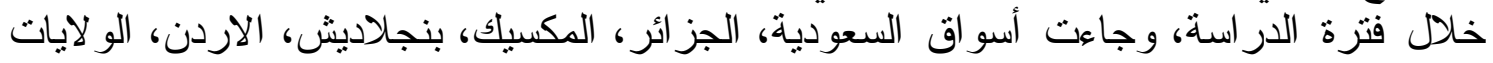

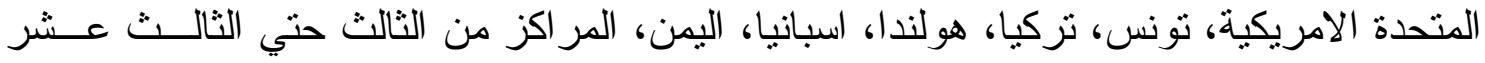

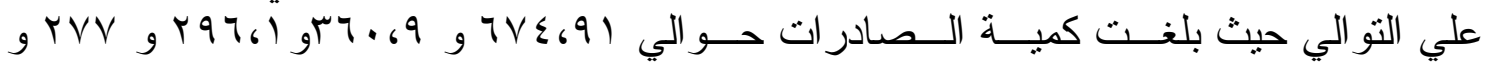

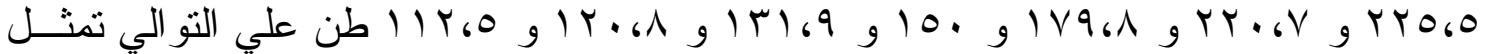
حـ و و

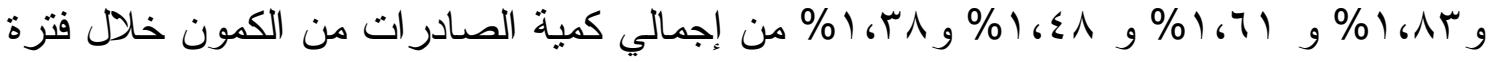

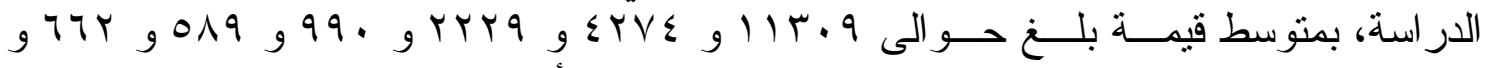

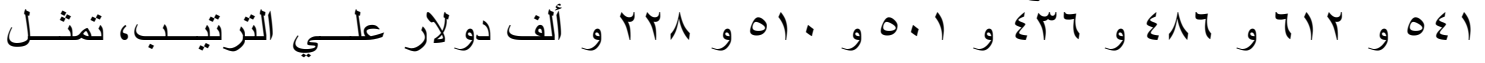


Website: www.aun.edu.eg/faculty_agriculture/journals_issues_form.php E-mail:ajas@aun.edu.eg

حو الي \%

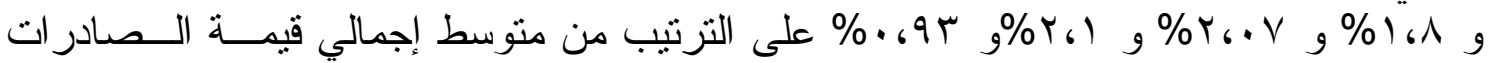

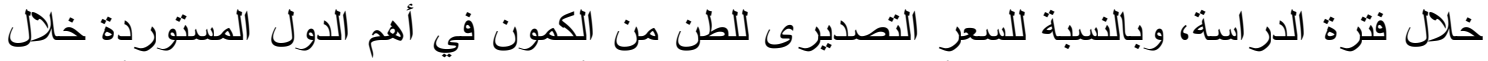

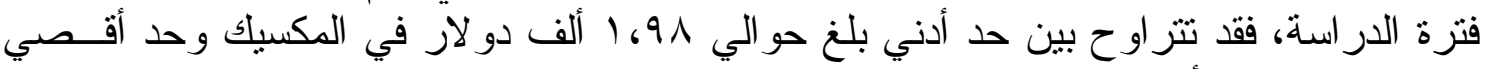

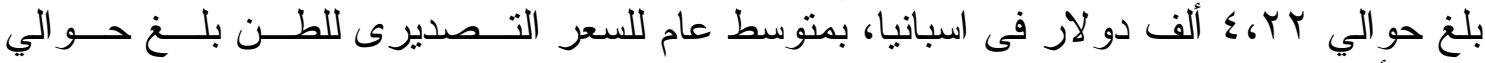

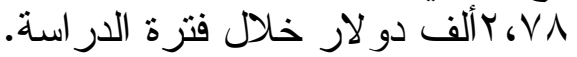

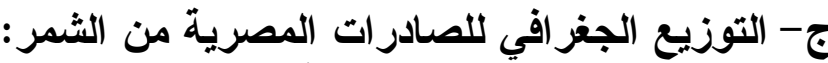

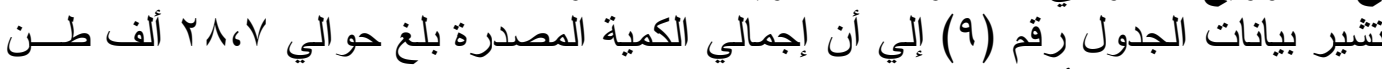

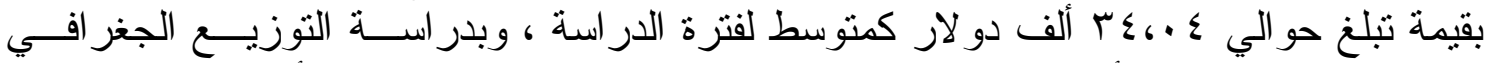

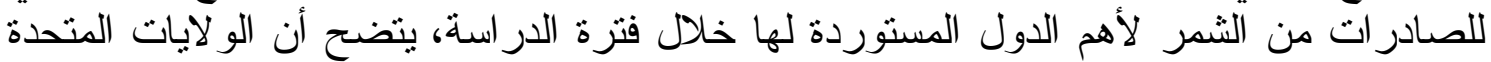

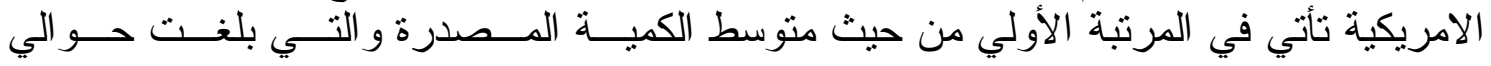

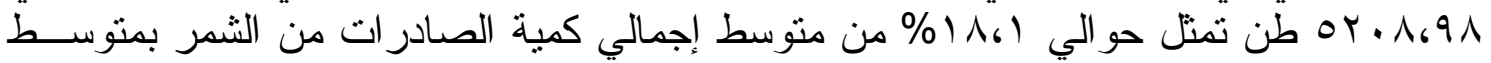

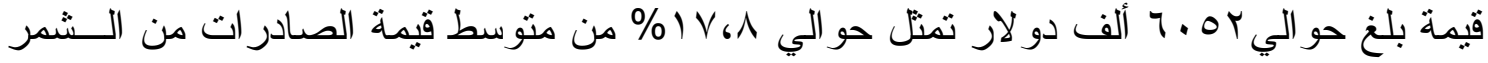

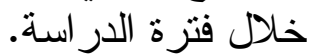

جدول رقم ^. التوزيع الجغرافي للصادرات من محصول الكمون إلي أهم الاول المستوردة لــه خـلهل

\begin{tabular}{|c|c|c|c|c|c|}
\hline \multicolumn{6}{|c|}{18} \\
\hline (ألف دولار) & $\%$ & 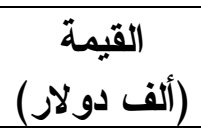 & $\%$ & الكمية & الاولة \\
\hline r.9T & $\sum 7,74$ & $11 r .9$ & $\left\{7_{6} \cdot \varepsilon\right.$ & TVฯฯ, & 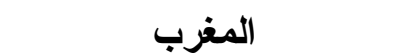 \\
\hline Y.TV & IVGTY & $\varepsilon r V \varepsilon$ & 10,97 & $15 \cdot 0,10$ & ليبيا \\
\hline$r_{6} r$. & 9619 & TrYq & A,ro & $7 V \varepsilon(9)$ & السعودية \\
\hline$Y_{6} V \varepsilon$ & $\varepsilon_{6} \cdot 1$ & 99. & $\varepsilon$ «) & 47.699 & الجز ائر \\
\hline 1.91 & $T_{6} \leqslant Y$ & 019 & & r97.. 9 & المكسيك \\
\hline r.ru & Y.VY & & r.ru & TVV & بنجلاديش \\
\hline r.ra & r,Tr & $0 \leqslant 1$ & r.Vo & rYO,O & الإردن \\
\hline T.VV & r.OY & TIT & r.79 & KT.GTV & الولايات المتحدة الامريكية \\
\hline r.V. & r & 纟八7 & r.19 & $1 \times 9680$ & تونس \\
\hline r.9) & 1.69 & हाч & l.Ar & 10. & تركيا \\
\hline$r_{6} v 9$ & $r_{6} \cdot V$ & 0.1 & 1,71 & 141.91 & هولندا \\
\hline E,TY & $T_{61}$. & 01. & $1, \varepsilon \wedge$ & $1 Y \cdot 6 V 4$ & اسبانيا \\
\hline$r_{6} .4$ & .694 & rMA & $1,4 \mathrm{~N}$ & $11 r_{60}$. & اليمن \\
\hline$r_{6} \leqslant 1$ & r.TV & 19. & $\varepsilon, 4 \Lambda$ & $r 01.07$ & اخري \\
\hline r.VA & $1 \cdots$ & rEYOV & $1 \ldots$ & $\Lambda 1 \Lambda .6 \mathrm{~V}$ & الجملة \\
\hline
\end{tabular}

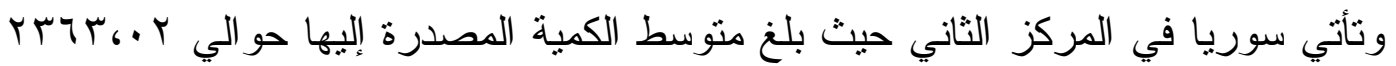

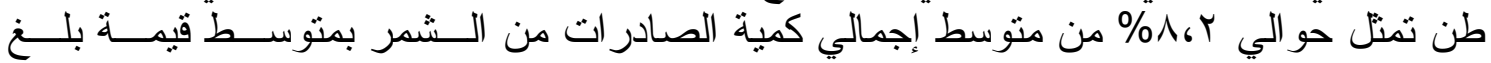

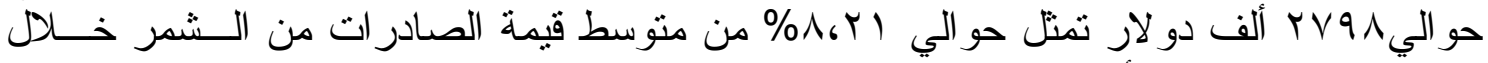

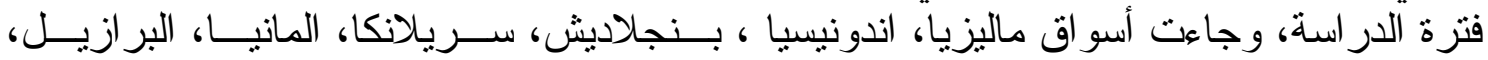

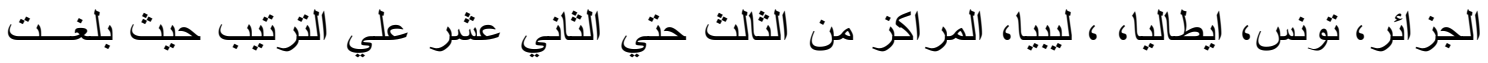




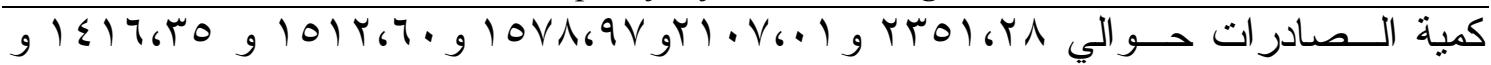

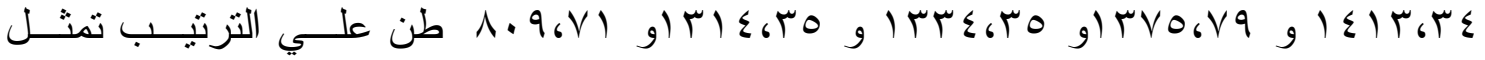

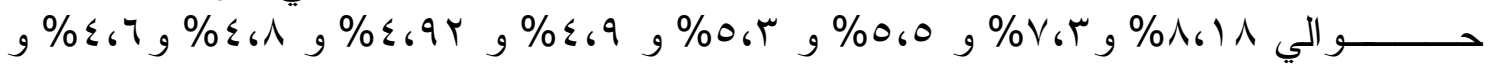

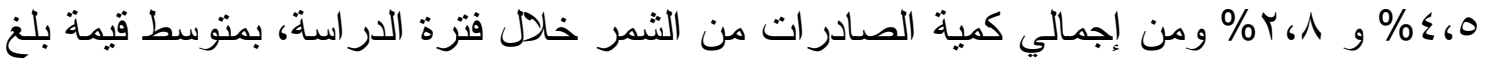
حــــ اللى ع

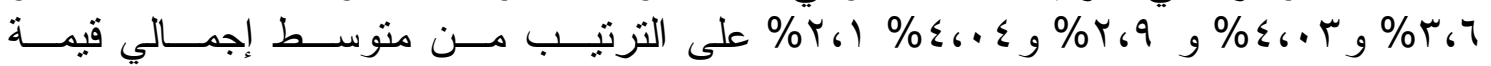

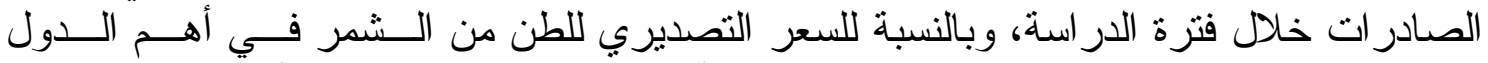

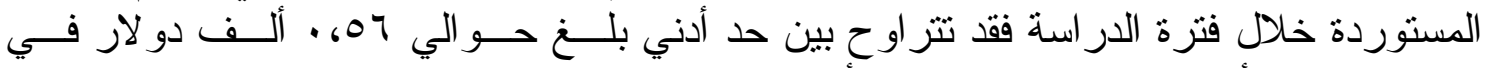

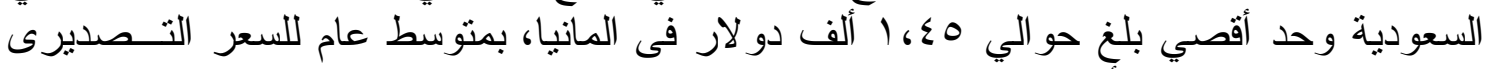

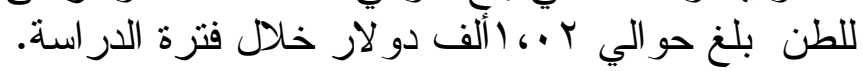

جدول رقم 9 ـ. التوزيع الجغرافي للصادرات من محصول الثمر إلي أهم الدول المستوردة لــهـ خـلهل

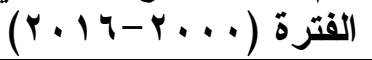

\begin{tabular}{|c|c|c|c|c|c|}
\hline (ألف دولار) & $\%$ & 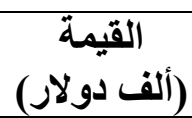 & $\%$ & الكمية & الدولة \\
\hline 1617 & $1 V_{6} A$ & $7.0 Y$ & $\left|1 \Lambda_{6}\right|$ & $0 Y \cdot \Lambda_{6} 91$ & الولايات المتحدة الامريكية \\
\hline 1,11 & $\Lambda_{6}, Y_{1}$ & $r \vee q \wedge$ & $\Lambda_{6} Y$ & TrצT6. & سوريا \\
\hline $1, Y Y$ & $\Lambda_{6} \varepsilon$ & 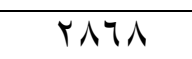 & $\left.\Lambda_{6}\right) \wedge$ & TMO1, TA & ماليزيا \\
\hline $1, \varepsilon \leqslant$ & $\Lambda_{6} \wedge$ & 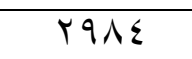 & $V_{6} T^{T}$ & $r_{1} \cdot V_{6} \cdot 1$ & اندونيسيا \\
\hline $1, r \Sigma$ & 7.4 & YIN & 0,0 & $10 \mathrm{VA} .9 \mathrm{~V}$ & بنجلاديش \\
\hline $1 . \mathrm{MV}$ & $76 \cdot \varepsilon$ & Y.OV & $0, \mathrm{~T}$ & $101 Y_{67}$ & سريلانكا \\
\hline $1, \leqslant 0$ & $76 \cdot r$ & $r \cdot \leqslant q$ & $\varepsilon 69$ & $1 \leqslant 17.10$ & المانيا \\
\hline $.6 \mathrm{AV}$ & $\Gamma_{6} T$ & ITH & \&.9Y & $1 \leqslant 1 \mu_{6} T \leqslant$ & البرازيل \\
\hline .699 & $\varepsilon_{6} \cdot r$ & ITVY & $\varepsilon_{61}$ & $14 \vee 06 V q$ & الجزائر \\
\hline $.6 \times 7$ & r.9 & 1.11 & $\varepsilon 67$ & 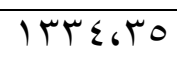 & تونس \\
\hline 16.0 & $\varepsilon_{6} \cdot \varepsilon$ & IrVo & $\varepsilon_{6} 0$ & $1 M 1 \leqslant 60$ & ايطاليا \\
\hline .619 & $\left.r_{6}\right)$ & $V Y \xi$ & $T_{6} \Lambda$ & $1.96 \vee 1$ & ليبيا \\
\hline $.6 \wedge \mathrm{V}$ & 1,11 & 097 & $Y_{6} \varepsilon$ & $7 \wedge \varepsilon_{6} \cdot \wedge$ & الاردن \\
\hline $.6 V Y$ & 1,4 & そ1 & 1.9 & OVT.7. & الهند \\
\hline .607 & .610 & $r \wedge \wedge$ & 1,1 & $01 \mu_{6} \mu_{V}$ & السعودية \\
\hline $16 \cdot 1$ & 165 & $\varepsilon \wedge V$ & $16 \mathrm{~V}$ & $\Sigma \wedge \Psi_{6}{ }^{\prime}$ & فرنسا \\
\hline .699 & 1,5 & $\varepsilon Y V$ & 1,0 & $\leqslant Y \wedge_{6} 70$ & هولندا \\
\hline .694 & 1.6 & r9o & 168 & $\varepsilon Y 0_{6} \cdot 1$ & المغرب \\
\hline .610 & .61 & roq & 16.7 & r.7.Yo & تركيا \\
\hline .690 & .610 & rq. & 16.0 & T. E, YO & باكستان \\
\hline $.6 V 1$ & .67 & $T \cdot V$ & 16.1 & Yq)، & جنوب افريقيا \\
\hline .695 & $.6 \vee \wedge$ & rדA & .691 & $r \wedge \varepsilon_{6} \cdot 1$ & الامارات \\
\hline .674 & $.6 \leqslant 7$ & 17. & $.6 \wedge 9$ & Y07.71 & بريطانيا \\
\hline 16.4 & .671 & TKE & .679 & YYA, Mo & النمسا \\
\hline .671 & $.6 \leqslant 0$ & 104 & $.6 \mathrm{VV}$ & YrT.qV & اسرائيل \\
\hline $1 . \leqslant 9$ & $96 \varepsilon$ & 19. & $\left.\Lambda_{6}\right)$ & 4111699 & اخري \\
\hline $16 \cdot r$ & $1 \ldots$ & $\Gamma \xi \cdot \varepsilon r$ & $1 \ldots$ & YAVY. .0 & الجملة \\
\hline
\end{tabular}


المصدر : وزارة الصناعة و التجارة الخارجية، الهيئة العامة للرقابة علي الصادرات و الو اردات، بيانات غير منشورة. الملخص و التوصيات

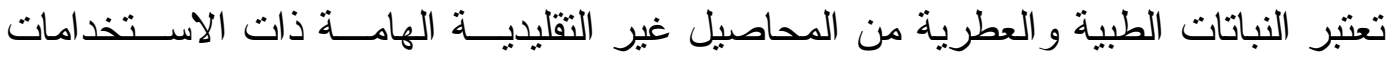

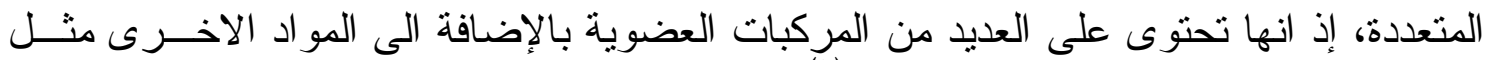

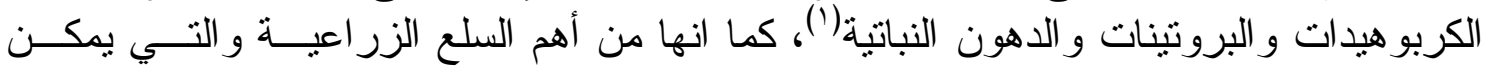

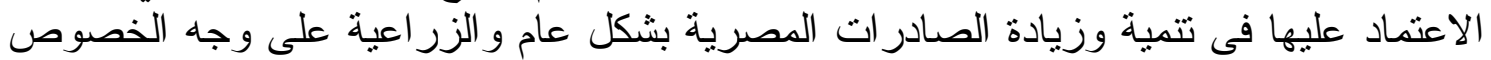

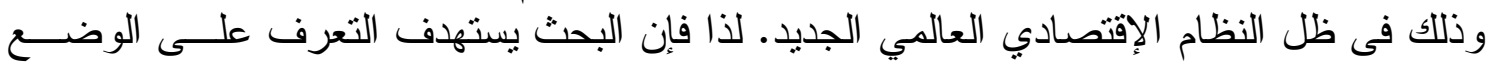

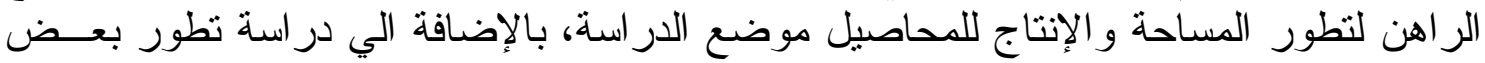

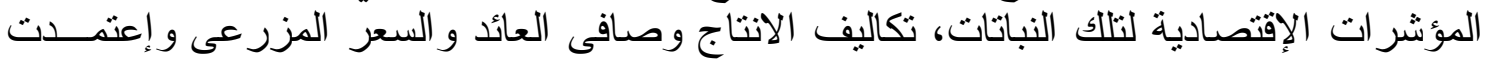

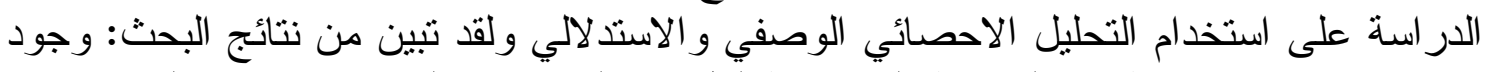

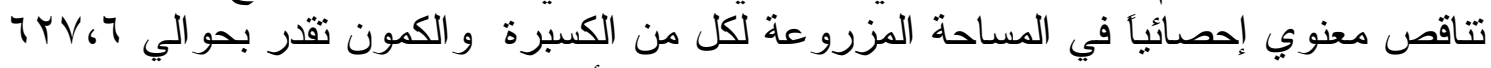

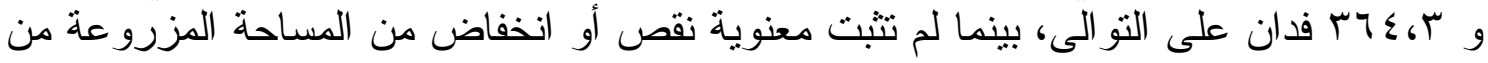

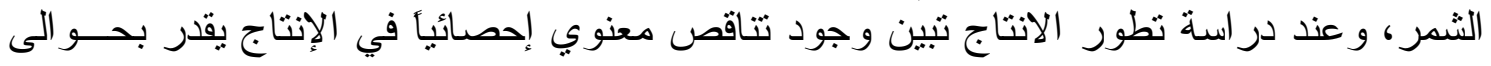

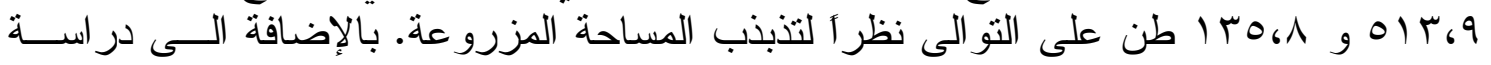

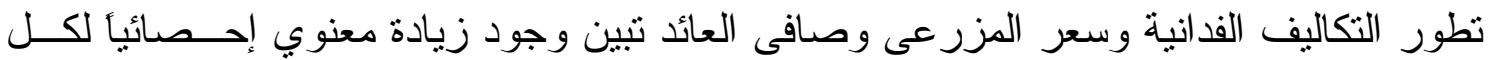

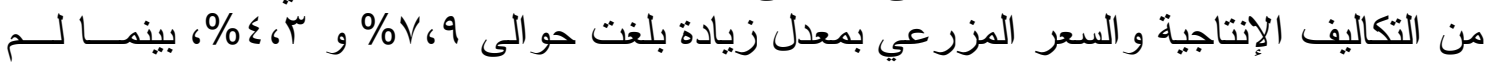

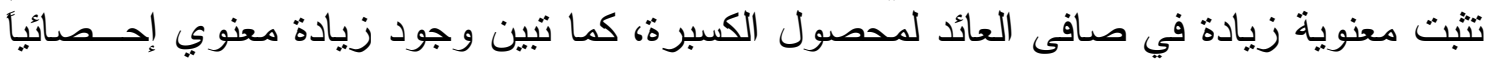

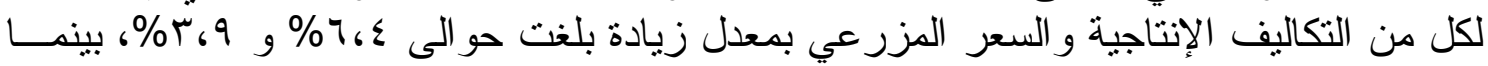

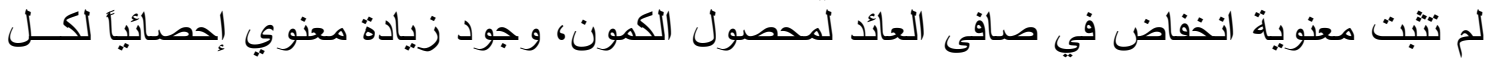

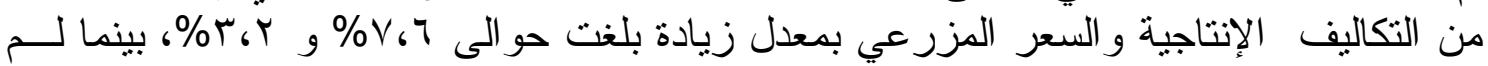

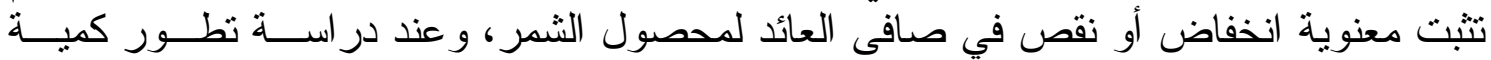

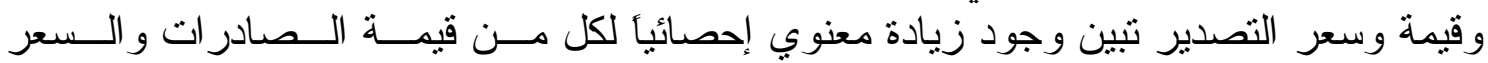

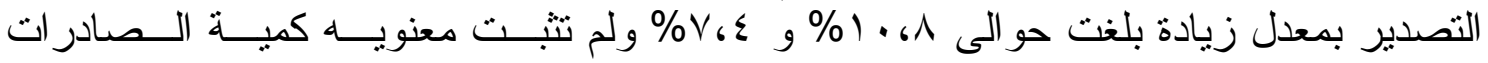

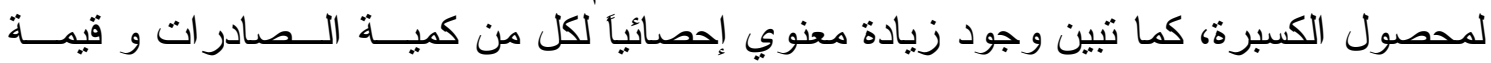

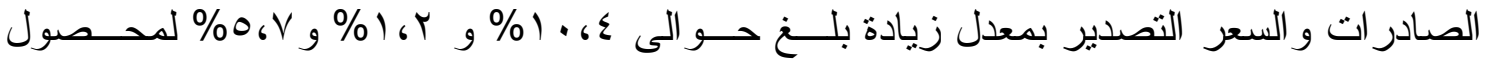

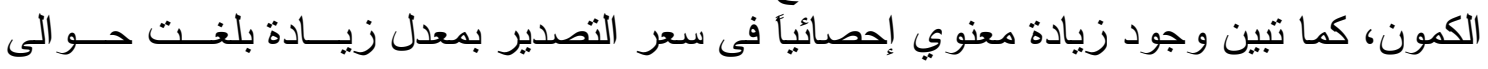

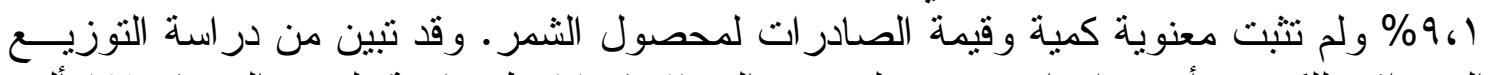

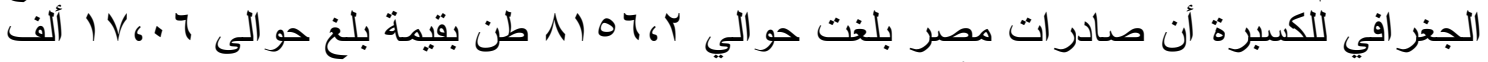

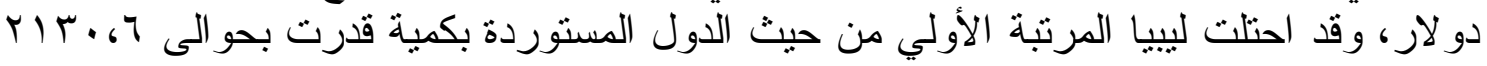

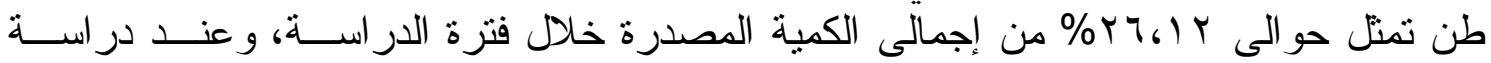

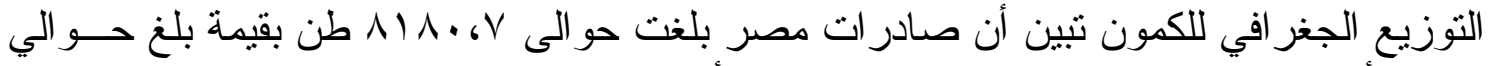

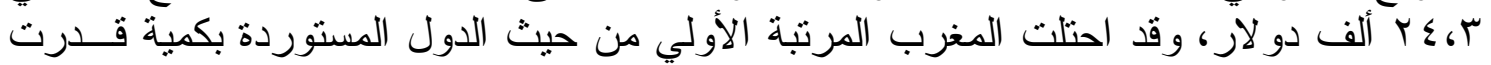

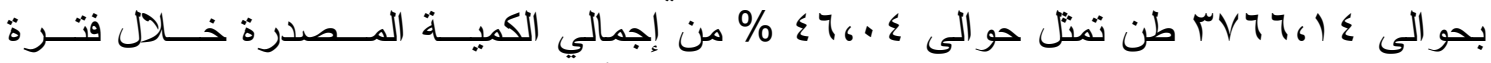

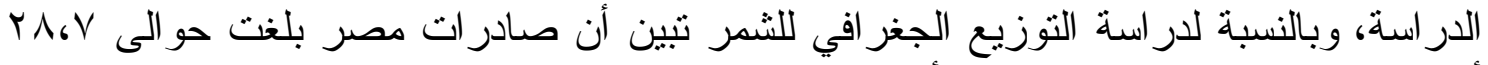

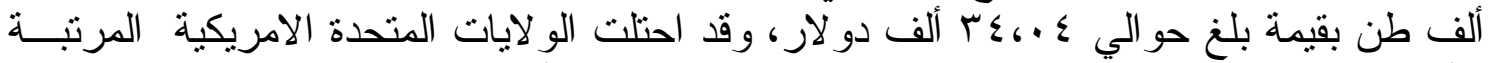

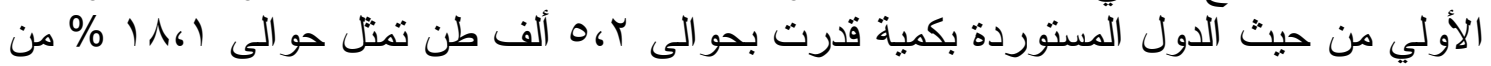

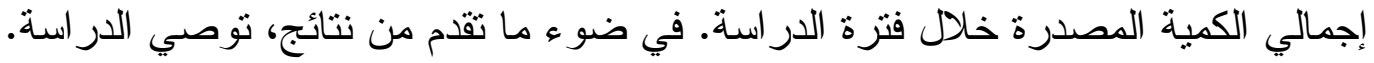

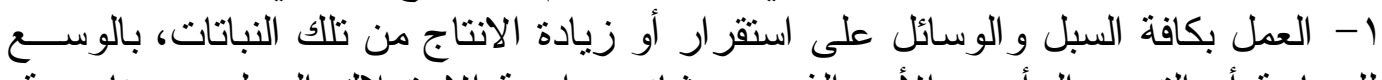

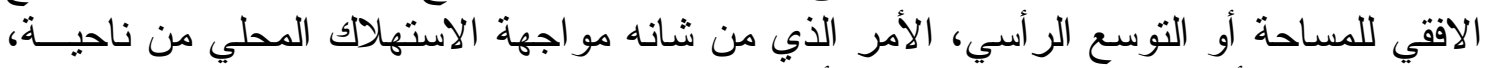
وتلبيةٌ طلبات الأسو اق الخارجية من الرية ناجية أخري. 


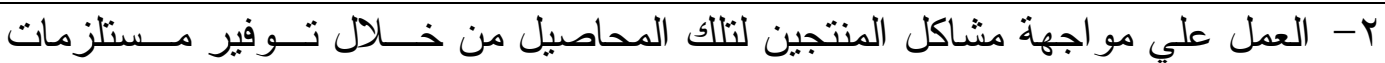
الانتاج من ناحية، وتفعيل دور الارشاد لتوعية المنتجين بالممارسات الزر اعية من ناحية أخري. r- إمداد المنتجين و المصدرين المصريين بالمعلومات و الإنباء عن الأسواق الخارجية.

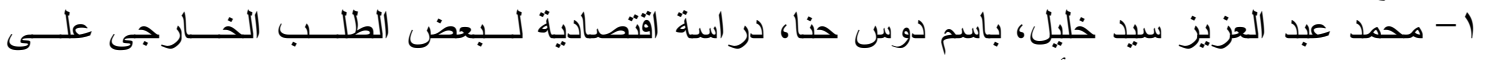

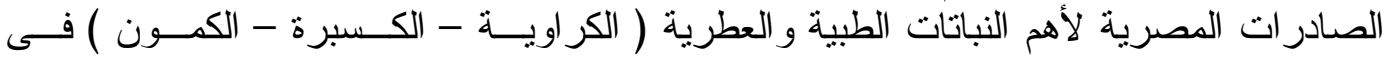

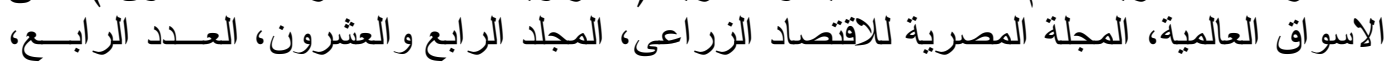

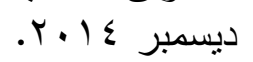

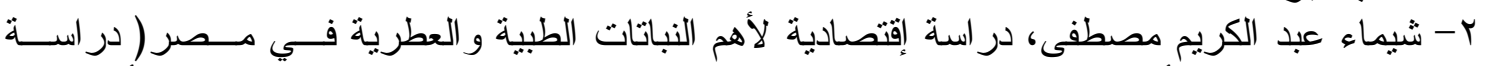

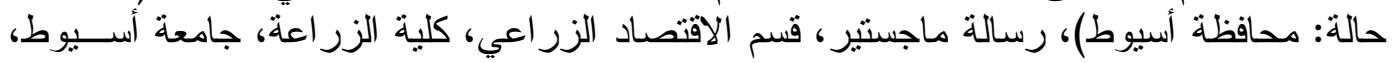

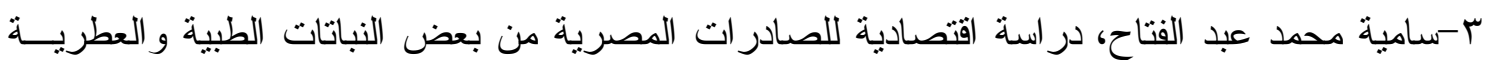

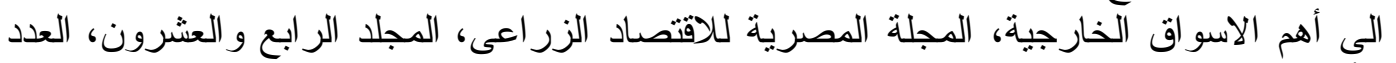

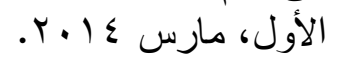

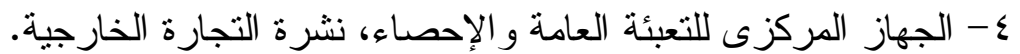

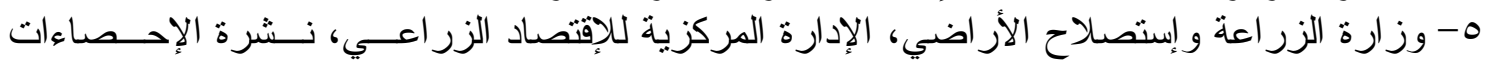

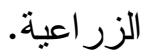




\title{
An Economic Study of the Productive Situation and Foreign Trade of the most Important Medicinal and Aromatic Plants in Egypt
}

\author{
Hassan M. Radwan
}

Higher Institute for Cooperation and Agricultural Extension in Assiut

\begin{abstract}
Medicinal and aromatic plants are important non-traditional crops of multiple uses, that it has several types of organic compounds in addition to other substances such as carbohydrates, proteins and vegetable fats so that the aim of this research is to identify the current status of the development of area and crops production under study, in addition to study of the development of some economic indicators of these plants, production costs, net yield and farm price. the results of the study showed that there was a statistically significant decrease in the cultivated area for both kaspera and cumin, estimated at 627.6 and 364.3 feddans respectively, and when studying the development of production, there was a significant statistical decrease in production estimated at 513.9 and 135.8 tons, respectively, due to the fluctuation of cultivated area, In addition to the study of the development of the cost of the feddan and the price of the farm and the net yield, there was a significant statistical increase for both production costs and agricultural price at an increase rate of about $7.9 \%$ and $4.3 \%$ there was also a statistically significant increase in both production costs and agricultural prices at an increase rate of about $6.4 \%$ and $3.9 \%$ there was a statistically significant increase in both production costs and agricultural prices at an increase rate of about $7.6 \%$ and $3.2 \%$. When examining the evolution of quantity, value and export price, there was a statistically significant increase in the value of exports and export prices at an increase rate of about $10.8 \%$ and $7.4 \%$. There was also a statistically significant increase in the quantity of exports, export value and export price at an increase rate of about $10.4 \%, 1.2 \%$ and $5.7 \%$ for cumin yield, there was also a statistically significant increase in the export price at an increase rate of about $9.1 \%$. A study of the geographical distribution of kasbera showed that Egypt's exports reached 8156.2 tons valued at about 17.06 thousand dollars. Libya ranked first in terms of importing countries with an estimated quantity of 2130.6 tons representing $26.12 \%$ of the total quantity exported during the study period. When studying the geographical distribution of the quintile, it was found that Egypt's exports amounted to about 8180.7 tons with a value of about 24,3 thousand dollars, Morocco ranked first in terms of importing countries with an estimated quantity of 3766.14 tons representing about $46.04 \%$ of the total quantity exported during the study period. As for the study of the geographical distribution of the fennel it was found that Egypt's exports amounted to about 28.7 thousand tons worth about 34.04 thousand dollars. The United States ranked first in terms of importing countries with an estimated quantity of about 5.2 thousand tons representing about $18.1 \%$ of the total quantity exported during the study period.
\end{abstract}

